\title{
Desempleo y criminalidad en los estados de la frontera norte de México: un enfoque espacial bayesiano de vectores auto- regresivos
}

\author{
Unemployment and crime in the Northern-border states of \\ Mexico: a spatial-Bayesian autoregressive vector approach
}

\section{Víctor Hugo Torres Preciado}

Recibido: 26 febrero 2016 / Aceptado: 14 julio 2016

\section{Resumen}

Ante los recientes episodios de intensificación en la actividad delictiva en México, llama la atención la escasez de estudios que analizan su relación con las fluctuaciones en la tasa de desempleo. El objetivo de esta investigación consiste en evaluar el impacto que tendría un desajuste que incremente sorpresivamente la tasa de desempleo en los estados de la frontera norte, sobre la actividad criminal, en particular respecto del número de robos. Con este fin, se aplica un VAR bayesiano espacial de acuerdo con la propuesta de LeSage y Cashell (2015). Los resultados sugieren que la respuesta del número de robos es heterogénea en los estados de la frontera norte de México. Por ejemplo: en algunos estados como Chihuahua, el número de robos parece responder de forma transitoria; en tanto que en Tamaulipas, este tipo de delito reacciona en menor medida a las fluctuaciones en su tasa de desempleo y la de sus estados vecinos.

Clasificación JEL: C33; E24; K42; R23.

Palabras clave: Desempleo. Criminalidad. Robos. Frontera Norte. México. Vector Autorregresivo Espacial Bayesiano.

\section{Abstract}

Despite the recent episodes of criminal activity intensification in Mexico, it is striking the scarcity of studies analyzing its relationship with unemployment rate fluctuations. The aim of this research is to evaluate the impact that an

\footnotetext{
*Universidad de Colima, Av. Josefa Ortiz de Domínguez no. 67, La Haciendita, Villa de Álvarez, Colima. C.P. 28970. Correo electrónico: torrespreciado@ucol.mx
} 
external shock of a surprising increase in unemployment rates would have upon crime activity, particularly, robberies in Mexico's northern Border States. To this end, a spatial Bayesian VAR that follows LeSage and Cashell (2015) is applied. Results suggest a heterogeneous response of robberies across northern Border States. For instance, in some states such as Chihuahua, the number of robberies seems to deploy transitory effects, while Tamaulipas appears to be less sensitive to own and neighbor state unemployment fluctuations.

JEL Clasification: C33; E24; K42; R23.

Keywords: Unemployment. Crime. Robbery. Northern Border. Mexico. Spatial Bayesian Vector Autoregression.

\section{Introducción}

Los fundamentos del análisis económico moderno de la criminalidad fueron establecidos por Becker (1968), quien con un enfoque microecómico caracterizó al acto delictivo como un problema de elección racional individual. Su contribución dio lugar a un cúmulo de estudios teóricos y empíricos que ayudaron a profundizar el conocimiento de la conducta criminal en ambientes de incertidumbre, y su reacción ante diferentes medidas de combate a la criminalidad (Ehrlich, 1973), (Witte, 1980), (Schmitd y Witte, 1984). No obstante, aunque el enfoque económico de la criminalidad propuesto por Becker (1968) ha sido ampliamente aceptado en el ámbito académico internacional y en el diseño de políticas de seguridad pública, aún es insuficiente para abordar algunos aspectos del vínculo entre actividad criminal y las condiciones económicas que prevalecen en países y regiones.

En este sentido, un conjunto de investigaciones se ha enfocado en analizar, desde una perspectiva principalmente agregada, el impacto que la actividad delictiva ejerce en diferentes medidas de desempeño económico nacional y regional. Por ejemplo, Zelekha y Bar-Efrat (2011) evalúan el efecto que la actividad delictiva tiene en la inversión privada en Israel. Daniele y Marani (2010), por su parte, analizan el impacto del crimen organizado en la inversión extranjera directa en Italia. Asimismo, algunos estudios han puesto su atención en analizar el efecto potencialmente distorsionante que la actividad delictiva local ejerce en la pequeña empresa (Krkoska y Robeck, 2009), (SBP, 2008). Paralelamente, un creciente número de estudios internacionales analizan el efecto en la tasa crecimiento económico (Neanidis y Papadopoulou, 2013; Kumar, 2013; Goulas y Zervoyianni, 2013; Öcal y Yildirim, 2010). 
Similarmente, el estudio acerca del efecto de las condiciones económicas sobre los diferentes tipos de actividad delictiva ha cobrado relevancia con el apoyo de enfoques metodológicos modernos, permitiendo revisar hipótesis inicialmente investigadas por criminólogos a principios del siglo veinte (Bonger, 1916; Thomas, 1925). Los estudios recientes en este ámbito reconocen que durante los episodios de recesión económica, el consiguiente aumento en la tasa de desempleo se convierte en un factor potencialmente detonante de la actividad delictiva; sin embargo, la evidencia empírica internacional dista de ofrecer un consenso en este sentido.

En México, la economía ha atravesado recientemente por episodios caracterizados por aumentos en la tasa de desempleo y la intensificación en la actividad delictiva. En el primer caso, la crisis económico-financiera internacional, que iniciara a principios de 2008 en Estados Unidos, ocasionó que la actividad económica agregada en México encarara contracciones de hasta diez por ciento, como ocurrió en el segundo trimestre de 2009 (Mendoza, 2013), con el consiguiente aumento en los despidos laborales. De hecho, en el periodo poscrisis, cuando la actividad económica agregada de Estados Unidos comenzaba a recuperarse, en algunos estados mexicanos como Baja California, la tasa de desempleo promedio aumentó poco más de cuatro puntos porcentuales, llegando a alcanzar hasta 7.6 por ciento. En particular, los estados ubicados en la frontera norte parecen haber enfrentado los mayores desajustes en el mercado laboral, ya que Coahuila y Chihuahua llegaron a alcanzar tasas de desempleo de 9.7 por ciento, mientras que en Nuevo León y Tamaulipas llegaron hasta 8.5 por ciento (Torres, 2013).

Con respecto a la actividad delictiva, entre los años 2005 y 2009, la tasa de delitos totales por cada cien mil habitantes aumentó poco más de diez por ciento, particularmente la tasa de robos, cuyo incremento fue cercano al veinticuatro por ciento. Esta situación sugiere no solamente que los costos económicos y sociales asociados habrían aumentado, sino también la presencia de una estructura de incentivos suficientemente afianzada para motivar la actividad criminal en el país. Por otro lado, debe mencionarse que la distribución espacial de la actividad delictiva dista de ser homogénea en México (Torres, Polanco y Venegas, 2015), de tal forma que algunos estados afrontarían costos económicos y sociales comparativamente mayores, al concentrar un mayor número de delitos. Tal sería el caso de la frontera norte de México, una región que agrupa a los estados de Baja California, Sonora, Chihuahua, Coahuila, Nuevo León y Tamaulipas, y que desde 2005 contribuye con aproximadamente el veinte por ciento del número total de delitos en el país (ver cuadro A2); en este sentido, el aumento observado en la tasa de desempleo pudiera haber exacerbado la actividad delictiva, principalmente aquellas de tipo adquisitivo, como los robos. 
De hecho, la situación de inseguridad pública que atraviesa México, ha motivado la aparición de un número creciente de investigaciones que pretenden abordar este fenómeno desde una perspectiva económica. Por ejemplo, entre los estudios recientes que abordan el análisis de los determinantes de la actividad criminal en México se encuentra el elaborado por Osorio (2012); también, las investigaciones de Feliz (2012), Pan et al. (2012), Enamorado (2013), González (2014) y Torres, Polanco y Tinoco (2015) que analizan el efecto de la criminalidad en el crecimiento económico a escala regional en el país. En el trabajo de Torres, Polanco y Venegas (2015), se evalúa el efecto de la criminalidad en la inversión privada regional y nacional, y en la investigación de Verdugo, Pedroni y $\mathrm{Hu}$ (2015), los autores evalúan el efecto de un choque a la tasa de homicidios en variables como el PIB y la IED mediante un modelo VAR estructural en panel. No obstante, llama la atención que dada la importancia que las variaciones observadas en la tasa de desempleo tendrían en el comportamiento de la actividad delictiva en México, el número de investigaciones que abordan su análisis aún es escaso, particularmente a escala regional.

En este contexto, el objetivo de esta investigación consiste en analizar el efecto que las variaciones en la tasa de desempleo tendrían sobre el comportamiento del número de robos en los estados de la frontera norte de México: una región donde recientemente se han presentado los aumentos más notorios en la tasa de desempleo y también en el número de delitos. Con este fin, se utiliza un modelo de vectores regresivos bayesiano que incorpora la interacción espacial entre los estados de la frontera norte, condicionada a su cercanía geográfica. En particular, se pretende responder si un choque que incremente la tasa de desempleo conduciría también a un incremento en el número de robos en los estados de la frontera norte del país.

El documento se organiza de la siguiente manera: en el segundo apartado, se revisa la literatura económica internacional y nacional que vincula la tasa de desempleo y la actividad criminal; en el tercer apartado, se explora el comportamiento del desempleo y el número de robos en los estados de la frontera norte de México; en el cuarto, se describe la metodología empleada $\mathrm{y}$, posteriormente, se realiza el análisis empírico; en el último apartado, se presentan las conclusiones.

\section{Revisión de la literatura}

El interés por profundizar en el efecto de las condiciones económicas adversas sobre la actividad delictiva, se encuentra en estudios de principios del siglo veinte. Entre estos, están los trabajos de Bonger (1916), Thomas (1925) y Phelps (1929), cuyo objetivo se centró en el efecto que las 
fluctuaciones en el corto plazo, de la tasa de desempleo agregada, tenían sobre diferentes indicadores de actividad criminal. Los estudios representaron los primeros esfuerzos por establecer relaciones empíricas con el apoyo de métodos estadísticos, siendo quizá el más exitoso, el trabajo de Phelps (1929), quien mediante la estimación de ecuaciones parabólicas encuentra una relación negativa.

No obstante la relevancia que el análisis de la relación entre la tasa de desempleo y criminalidad representa para el diseño de políticas económicas y sociales, su estudio se debilitó durante los siguientes treinta años. Posteriormente, se observa un resurgimiento a mitad de la década de los sesenta con los trabajos de Smigel-Leibowitz (1965), Fleisher (1966), y Ehrlich (1967). Sin embargo, el trabajo de Becker (1968), quien elabora un modelo microeconómico para estudiar la conducta criminal individual como resultado de una estructura de incentivos, basada en castigos y beneficios, sentó las bases para estudiar de forma sistemática, mediante la oferta de crimen, los determinantes de la actividad criminal. Aunque el trabajo de Becker (1968) representa un parte aguas en el análisis económico de la criminalidad, es la investigación de Ehrlich (1973), la que con base en el enfoque propuesto por Becker (1968), incluye de forma sistemática la relación entre desempleo y criminalidad.

En su análisis, Ehrlich (1973) establece la decisión de un individuo que elige la dotación de tiempo que destinará a actividades legales $(l)$ e ilegales $(i)$, con base en la probabilidad de ser aprendido $(p)$ o no (1-p), y asocia a esta decisión la probabilidad de ser desempleado $(u)$ o no $(1-u)$. Sus resultados indican que una probabilidad relativamente más alta de ser desempleo conduce a la decisión de delinquir. Lo que a escala agregada implica una relación positiva entre la tasa de desempleo y la actividad criminal, principalmente en actividades delictivas de tipo adquisitivo.

Diferentes estudios se han elaborado con base en las propuestas de Becker (1968) y Ehrlich (1973). Por ejemplo, Witte (1980) utiliza datos individuales de prisioneros liberados para estimar el modelo estándar de Becker (1968), y encuentra que la certeza y severidad del castigo influye significativamente en reducir la participación de los individuos en actividades delictivas, pero que salarios más elevados tendrían un efecto débil. Myers (1983), por su parte, utilizando bases de datos diferentes encuentra que la certidumbre y salarios más elevados reducirían la participación en actividades ilegales, pero la severidad del castigo tendría un efecto débil. Más recientemente, Cornwell y Trumbull (1994) estiman un modelo panel de oferta de crimen con datos para Estados Unidos. Los autores encuentran que los esfuerzos policiales son pequeños en comparación con otros estudios, pero que los salarios contribuyen a disminuir la decisión de delinquir. 
En esta línea de análisis, Yamada (1985) señala que los estudios elaborados a partir del enfoque de oferta del crimen, propuesto por Becker (1968) y Ehlrich (1974), proveen evidencia empírica que contrasta con respecto al signo y significación estadística encontrada cuando se analiza el vínculo tasa de desempleo-actividad criminal. El autor enfatiza que los estudios frecuentemente excluyen el efecto retardado que la tasa de desempleo puede ejercer en la actividad criminal a través del tiempo y, por otro, que las especificaciones empíricas suelen presentar multicolinealidad. En este sentido, los trabajos de Yamada (1985) y Yamada, Yamada y Kang (1991) se pueden considerar pioneros en la aplicación de metodologías modernas de series de tiempo al análisis de la relación de la tasa de desempleo y criminalidad, particularmente, a partir de vectores autorregresivos.

Por otro lado, un estudio que contribuyó a revitalizar el interés por investigar la relación entre tasa desempleo y actividad criminal es el elaborado por Cantor y Land (1985). Los autores señalan que los hallazgos empíricos que apuntan hacia una falta de consenso acerca de la relación entre ambas variables, obedece a la elaboración de teorías incompletas que fallan en reconocer la presencia no excluyente de dos efectos simultáneos: el efecto de oportunidad y el efecto de motivación. El primero explicaría la presencia de una relación negativa y contemporánea, debido a que en épocas de bajo desempleo, los individuos estarían más expuestos a robos u otro tipo de crimen adquisitivo. Por su parte, el segundo efecto hace referencia a una relación positiva y retardada en el tiempo, que resulta cuando las carencias motivan al individuo a delinquir ${ }^{1}$.

El hecho de proveer una explicación analítica, cuando los resultados empíricos sugieren relaciones contrapuestas, es parte del atractivo que ha hecho popular la propuesta de Cantor y Land (1985). Entre los estudios que abordan el análisis de la relación entre desempleo y actividad criminal bajo esta perspectiva, se encuentra el elaborado por Chiricos (1987), quien encuentra una relación positiva entre tasa de desempleo y crímenes a la propiedad, con datos en sección cruzada. Los trabajos de Smith, Devine y Sheley (1992), y Britt (1997), quienes utilizan datos en series de tiempo, encuentran evidencia a favor de ambos efectos. Sin embargo, algunos estudios ofrecen resultados diferenciados cuando utilizan datos en panel y especificaciones alternativas a la propuesta por Cantor y Land (1985). Por ejemplo, Levitt (2001) encuentra un efecto de motivación estadísticamente significativo cuando se consideran crímenes a la propiedad, pero no significativo cuando se vincula a crímenes violentos. Recientemente, Phillips

\footnotetext{
${ }^{1}$ Los efectos de oportunidad y de motivación propuestos por Cantor y Land (1985) se fundamentan, en el primer caso, en postulados de la teoría criminológica y, en el segundo, en el enfoque sociológico de la teoría de las actividades rutinarias.
} 
y Land (2012) revisaron las hipótesis iniciales de Cantor y Land (1985) utilizando una base de datos con 400 de los municipios más grandes en Estados Unidos, así como para su agregación a escala estatal y nacional. Los autores sostienen que las hipótesis inicialmente propuestas, se corroboran en los tres niveles de agregación, en 78 de 84 relaciones estimadas.

Adicionalmente, algunos estudios recientes sugieren que las ideas originales de Cantor y Land (1985) se abordan adecuadamente mediante la aplicación de metodologías que identifican la sincronización entre el ciclo económico y de criminalidad. En este sentido se encuentran los trabajos de Detotto y Otranto (2012), y de Cook y Watson (2014), quienes estudian el comportamiento cíclico de variables económicas adicionales a la tasa de desempleo.

En el caso de México, los estudios que abordan el efecto de la tasa de desempleo en algún tipo de actividad delictiva son escasos. Por ejemplo, el trabajo de Osorio (2012) evalúa el efecto que diferentes variables económicas, sociales y culturales ejercen en la tasa de homicidios en México. Sus resultados sugieren que la tasa de desempleo tiene un efecto positivo y estadísticamente significativo. En contraparte, diversos estudios elaborados recientemente se han enfocado principalmente en evaluar el efecto que la actividad delictiva ha tenido en algunas variables de desempeño económico.

Al respecto, la investigación de Feliz (2012) representa el primer intento por estudiar sistemáticamente el efecto de la actividad criminal en el crecimiento económico de México. Mediante la estimación de un modelo de convergencia condicional, el autor encuentra que un incremento del diez por ciento en la tasa de homicidios, se asocia con una disminución del 0.13 por ciento en la tasa de crecimiento. Por su parte, Pan et al. (2012), estiman un modelo espacial en panel de datos, y encuentran que los homicidios no ejercen un efecto significativo en la tasa de crecimiento económico regional de México. Por otro lado, en un estudio similar pero a escala municipal, Enamorado et al. (2013), encuentran evidencia de efectos negativos.

González (2014) analiza la relación de diferentes delitos del fuero común y federal en el crecimiento económico regional, sus resultados sugieren un efecto pequeño pero significativo en delito, que el autor denomina de alto impacto (homicidios, robos y delitos dolosos). Recientemente, Torres, Polanco y Tinoco (2015) estiman un modelo en panel de datos denominado Durbin espacial, para analizar el efecto de la actividad delictiva en el crecimiento económico regional de México. Sus hallazgos indican que la tasa de homicidios y robos ejercen un efecto negativo y significativo en el crecimiento económico regional, además encuentran que la presencia de efectos de difusión interregionales refuerzan su efecto negativo. 


\section{Desempleo y criminalidad en la frontera norte de México}

La actividad delictiva en México ha experimentado recientemente un periodo de intensificación que ha obligado a las autoridades gubernamentales a priorizar el restablecimiento de la seguridad pública, mediante reformas al Sistema Nacional de Seguridad Pública (Torres y Polanco, 2014). En particular, el número total de delitos del fuero federal se incrementó veintidós por ciento durante el periodo entre 2005 y 2011, alcanzando en este último año la mayor cifra en la última década con 1,729,934 delitos (cuadro A2). Sin embargo, la actividad delictiva parece iniciar un declive al disminuir ocho por ciento la cifra agregada de delitos entre 2011 y 2014.

Por su parte, el comportamiento de la actividad delictiva en la región frontera norte $^{2}$ del país, parece compartir una dinámica similar. En el periodo de expansión nacional de la actividad delictiva, los estados de la frontera en su conjunto experimentaron un incremento absoluto del 9.4 por ciento en el número de delitos totales, y similarmente al agregado nacional, una disminución del 8.8 por ciento entre 2011 y 2014. No obstante, el pico más alto en el número de delitos totales en esta región ocurrió en 2008, cuando alcanzó la cifra de 390,000 delitos totales, que al considerarse como referencia, implica una disminución del catorce por ciento hacia el año 2014 (cuadro A2).

En conjunto, la región frontera norte ha contribuido de forma significativa a la actividad delictiva nacional. En particular, en 2005, los estados de la frontera norte representaron el $23.6 \%$ de la actividad delictiva total en el país, y para el año 2015, esta contribución fue bastante similar, con $21.6 \%$ (cuadro A2). Al interior de esta región, algunos estados han experimentado una mayor incidencia delictiva. Por ejemplo, en 2015, el estado de Baja California contribuyó con el treinta por ciento de los delitos totales en la frontera norte, seguido cercanamente por Chihuahua y Tamaulipas con 20\% y $15.7 \%$, respectivamente. Para 2015, algunos estados preservaron su preponderancia en el conjunto de la actividad delictiva en la frontera norte, como Baja California y Chihuahua, cuyas contribuciones alcanzaron un treinta y dos y diecinueve por ciento, cada uno. El caso de Coahuila llama la atención, debido a que su posicionamiento en la actividad delictiva fronteriza ha aumentado significativamente al transitar de $7.5 \%$ en 2005 a $14.7 \%$ en 2015 (cuadro A3).

Con respecto al número de robos en la frontera norte del país, se observa que el pico de la actividad asociada a este delito ocurre en 2011, cuando la cifra

\footnotetext{
${ }^{2}$ En esta investigación, la región frontera norte está conformada por los estados de Baja California, Sonora, Chihuahua, Coahuila, Nuevo León y Tamaulipas.
} 
agregada fue de 207,547. A partir de este año, el número de robos ha disminuido hasta alcanzar una cifra de 132,895, para representar una contracción del treinta y seis por ciento, entre 2011 y 2014 (cuadro A4). El estado que más contribuye al conjunto de esta actividad delictiva en la frontera norte es Baja California, cuya participación ha aumentado de treinta y cuatro por ciento en 2005, hasta alcanzar treinta y nueve por ciento en 2015. Los estado de Sonora, Chihuahua y Nuevo León han reducido su participación en el mismo periodo, sin embargo, Tamaulipas y Coahuila la aumentaron, principalmente este último estado, al pasar de $6.3 \%$ a $11.5 \%$ (cuadro A5).

En la gráfica 1, se aprecia el comportamiento conjunto de ambas variables en cada uno de los seis estados de la frontera norte, para los datos mensuales estandarizados. En particular, se observa que en el caso de los estados de Chihuahua, Coahuila y Nuevo León, los periodos cuando la tasa de desempleo ha aumentado y descendido, parecen estar asociados a periodos similares de intensificación y desintensificación en la incidencia de robos. Sin embargo, esta relación no es tan clara cuando se visualizan las fluctuaciones mes a mes, donde frecuentemente se observa una asociación inversa. La disminución en ambas variables explicaría la reducción significativa que Chihuahua y Nuevo León han experimentado en sus contribuciones relativas, por ejemplo, en el caso del número de robos totales en la región frontera norte.

Por su parte, en el estado de Baja California, el patrón de asociación es aún menos claro, por ejemplo, la tasa de desempleo en Baja California parece descender lentamente desde finales de 2012, en tanto que el número de robos desciende con mayor rapidez. Por otro lado, el número de robos se incrementó incluso durante el periodo que prevaleció un bajo desempleo. En Sonora y Tamaulipas, el número de robos parece responder positivamente ante las variaciones en la tasa de desempleo, si bien se observa que en ambos estados la tasa de desempleo se ha mantenido relativamente estable (gráfica 1).

Finalmente, en el cuadro 1, se puede apreciar un resumen de tasa de desempleo y el número de robos en la frontera norte a partir de sus estadísticas básicas. En particular, se aprecia que el estado de Coahuila tuvo en promedio la tasa de desempleo más elevada, seguido por los estados de Tamaulipas y Nuevo León. Por su parte, el estado de Baja California tuvo el promedio más bajo en este indicador, debido a que a principios del periodo de estudio presentó las tasas de desempleo más bajas en la frontera norte e incluso en el país. En adición, el estado de Coahuila también ha experimentado la tasa de desempleo más elevada en el periodo entre los estados de la frontera norte, alcanzando un 9.6\%, aunque Chihuahua también 
ha atravesado por periodos similares de elevado desempleo. En contraparte, el estado de Baja California ha observado la tasa de desempleo más baja con $1.1 \%$ e incluso Chihuahua ha presentado una tasa mínima de $1.9 \%$, lo cual es congruente con la desviación estándar que sugiere una importante dispersión en este indicador. Con respecto al número de robos, el promedio más elevado durante el mismo periodo corresponde a Baja California, seguido por Chihuahua, aunque a una distancia notable. Similarmente, el máximo número de robos está registrado en Baja California con 7,064, en tanto que el mínimo número delitos corresponde a Sonora, y con un nivel similar a Coahuila (cuadro 2).

\section{Gráfica 1}

\section{Evolución de la tasa de desempleo y el número de robos en los estados de} la frontera norte de México 2005-2015 (datos estandarizados)
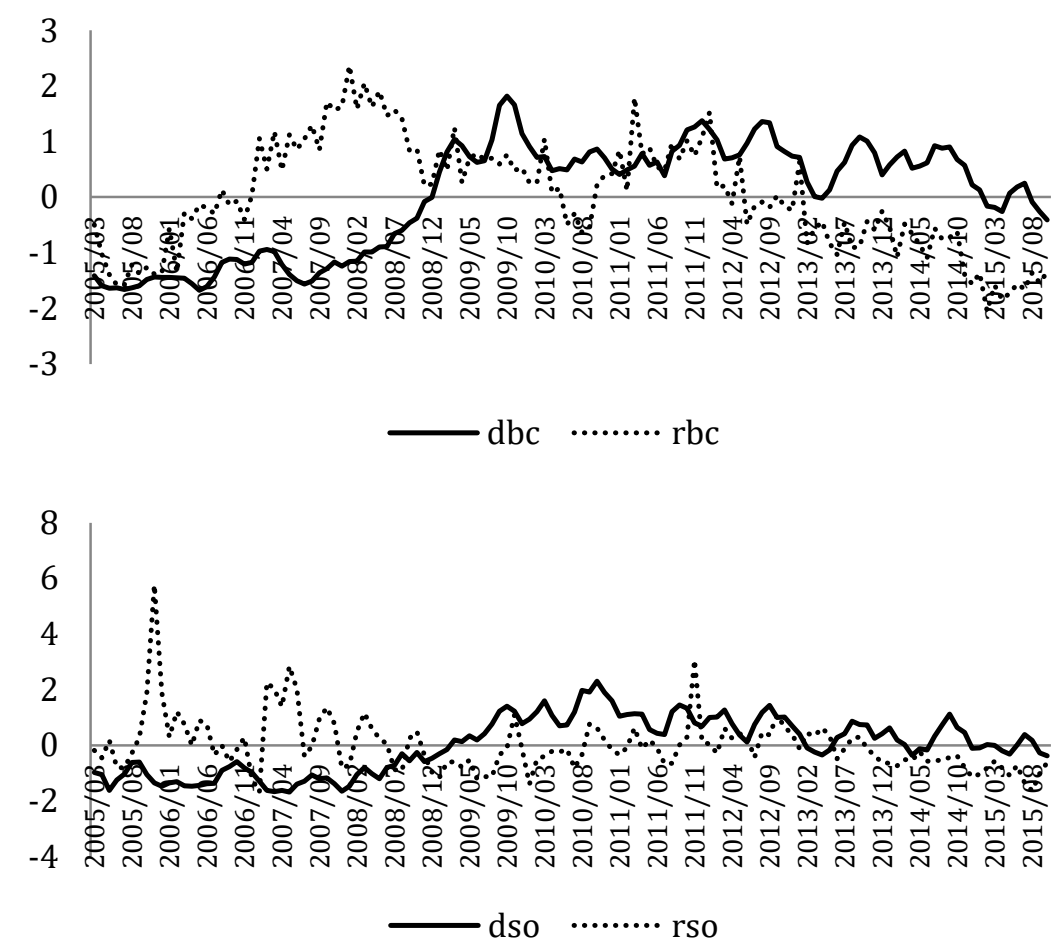

Fuente: elaboración propia con información del INEGI y el Secretariado Ejecutivo del Sistema Nacional de Seguridad Pública. La información abarca desde marzo de 2005 hasta octubre de 2015. La notación para el nombre de las variables, es: dbc, dso, dch, dcoa, dnl y dta, que son las tasas de desempleo para los estados de Baja California, Sonora, Chihuahua, Coahuila, Nuevo León y Tamaulipas. Asimismo, rbc, rso, rch, rco, rnl y rta representan el número de robos totales para los mismos estados. Nota: se estandarizaron los datos para facilitar la comparación de ambas series, lo que consiste en restar la media y posteriormente dividir entre la desviación estándar cada observación. 
Evolución de la tasa de desempleo y el número de robos en los estados de la frontera norte de México 2005-2015 (datos estandarizados)
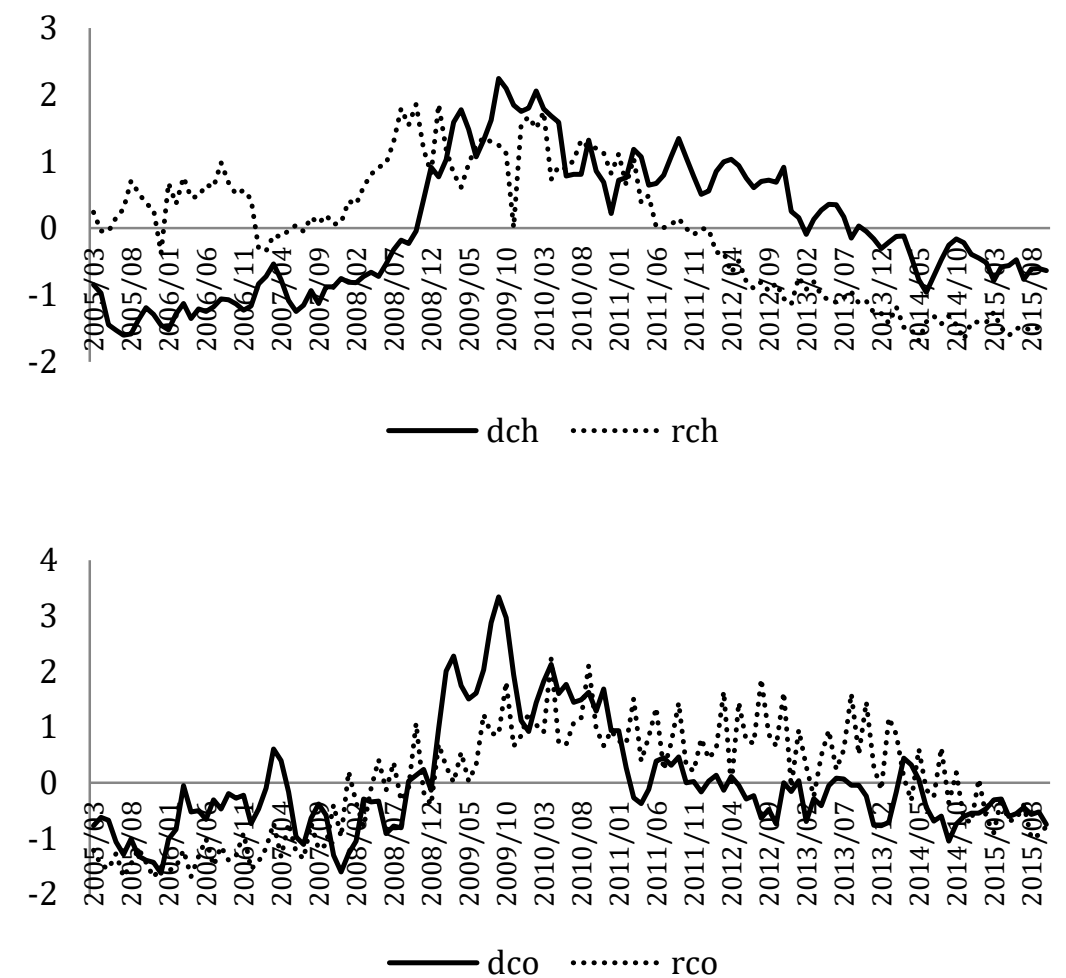

Fuente: elaboración propia con información del INEGI y el Secretariado Ejecutivo del Sistema Nacional de Seguridad Pública. La información abarca desde marzo de 2005 hasta octubre de 2015. La notación para el nombre de las variables, es: dbc, dso, dch, dcoa, dnl y dta, que son las tasas de desempleo para los estados de Baja California, Sonora, Chihuahua, Coahuila, Nuevo León y Tamaulipas. Asimismo, rbc, rso, rch, rco, rnl y rta representan el número de robos totales para los mismos estados. Nota: se estandarizaron los datos para facilitar la comparación de ambas series, lo que consiste en restar la media y posteriormente dividir entre la desviación estándar cada observación. 
Gráfica 1 (continuación)

Evolución de la tasa de desempleo y el número de robos en los estados de la frontera norte de México 2005-2015 (datos estandarizados)
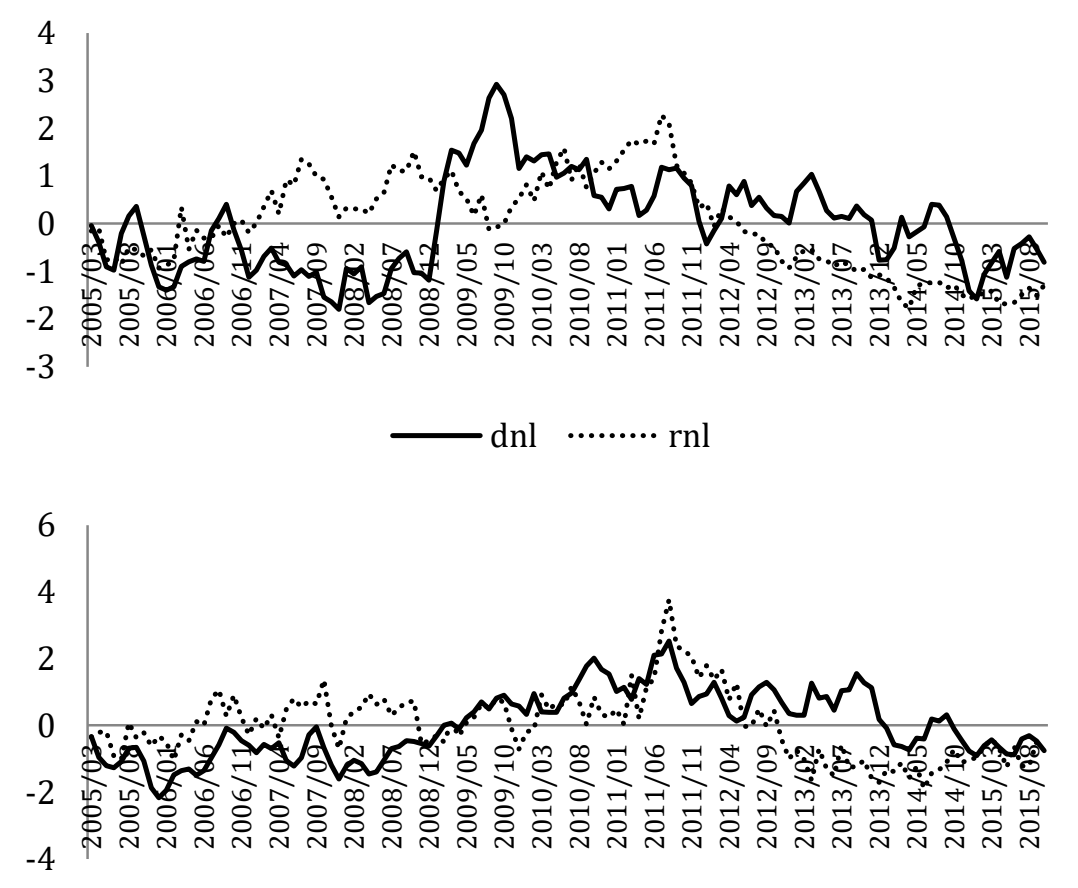

$\longrightarrow$ dtam $\cdots \cdots \cdot \cdot$ rtam

Fuente: elaboración propia con información del INEGI y el Secretariado Ejecutivo del Sistema Nacional de Seguridad Pública. La información abarca desde marzo de 2005 hasta octubre de 2015. La notación para el nombre de las variables, es: dbc, dso, dch, dcoa, dnl y dta, que son las tasas de desempleo para los estados de Baja California, Sonora, Chihuahua, Coahuila, Nuevo León y Tamaulipas. Asimismo, rbc, rso, rch, rco, rnl y rta representan el número de robos totales para los mismos estados. Nota: se estandarizaron los datos para facilitar la comparación de ambas series, lo que consiste en restar la media y posteriormente dividir entre la desviación estándar cada observación. 
Cuadro 2

Estadísticas básicas para la tasa de desempleo y el número de robos en los estados de la frontera norte de México 2005-2015

\begin{tabular}{lcccccccccccc}
\hline Estadístico & dbc & dson & dchi & dcoa & dnl & dtam & rbc & rson & rchi & rcoa & rnl & rtam \\
\hline Mediana & 5.2 & 5.1 & 4.8 & 5.6 & 5.5 & 5.5 & 5177 & 976.5 & 2522 & 1493 & 2406 & 1842 \\
Media & 4.4 & 5.1 & 5.1 & 5.8 & 5.5 & 5.7 & 5196 & 1007 & 2473 & 1455 & 2407 & 1849 \\
D.E. & 1.9 & 1.5 & 2 & 1.1 & 1 & 1.3 & 790.9 & 211.8 & 754.5 & 467.1 & 802.2 & 275.5 \\
D.E./ Media \% & 44 & 30 & 38.9 & 19.5 & 17 & 23 & 15.2 & 21 & 30.5 & 32.1 & 33.3 & 14.9 \\
Máximo & 7.9 & 8.6 & 9.5 & 9.6 & 8.3 & 9 & 7064 & 2229 & 3873 & 2494 & 4232 & 2887 \\
Mínimo & 1.1 & 2.5 & 1.9 & 4 & 3.8 & 2.8 & 3573 & 650 & 1201 & 658 & 988 & 1328 \\
Obs. & 128 & 128 & 128 & 128 & 128 & 128 & 128 & 128 & 128 & 128 & 128 & 128 \\
\hline
\end{tabular}

Fuente: elaboración propia con información del INEGI y el Secretariado Ejecutivo del Sistema Nacional de Seguridad Pública. La información abarca desde marzo de 2005 hasta octubre de 2015. La notación para el nombre de las variables, es: dbc, dson, dchi, dcoa, dnl y dtam, que son las tasas de desempleo para los estados de Baja California, Sonora, Chihuahua, Coahuila, Nuevo León y Tamaulipas, respectivamente. Asimismo, rbc, rson, rchih, rcoa, rnl y rtam representan el número de robos para los mismos estados.

\section{Aspectos metodológicos}

LeSage y Krivelyova (1999) señalan que la utilización de vectores autorregresivos en el análisis regional tiene dos limitaciones: 1) dada la presencia de retardos temporales en los vectores autorregresivos, con frecuencia se requiere contar con un amplio número de observaciones para estimar los parámetros también numerosos, y 2) la presencia de retardos en las variables independientes suele acompañarse de un elevada correlación que conduce a una degradación en la precisión de los parámetros estimados. Para solventar este problema, LeSage y Krivelyova (1999) sugieren utilizar la aplicación de métodos bayesianos en la estimación de los vectores autorregresivos con base en la propuesta de Doan, Litterman y Sims (1984), y Litterman (1986), quienes sugieren incorporar información bayesiana $a$ priori.

El enfoque bayesiano considera incierta la verdadera estructura de la población, y sin asignar un peso importante a alguno de los parámetros del modelo, aborda esta incertidumbre bajo la forma de una distribución de probabilidades con media y desviación estándar designados a priori, para cada uno de los parámetros del modelo. Si la información a priori es alterada por la "señal" y no por el "ruido" en las observaciones, entonces el problema de sobreajuste se reducirá notablemente (Ciccarelli y Rebucci, 2003).

Sin embargo, LeSage y Krivelyova (1999) señalan que la información bayesiana a priori que sugieren Doan, Litterman y Sims (1984), con respecto 
a la media y desviación estándar de los parámetros a estimar en un VAR, también conocida como información a priori de Minnesota, es restrictiva debido a que otorga un tratamiento similar a todas las variables con excepción de las variables dependientes rezagadas, lo cual es inadecuado cuando en el análisis se incorporan unidades espaciales que pueden presentar algún tipo de asociación. En este sentido, se espera que las unidades espaciales más cercanas ejerzan una influencia mayor que aquellas unidades espaciales más lejanas.

Con la finalidad de estudiar la relación entre desempleo y criminalidad en los estados ubicados en la frontera norte de México, se propone implementar un vector autorregresivo bayesiano (BVAR) conforme a la propuesta de LeSage y Cashell (2015), quienes utilizan un enfoque híbrido que combina el enfoque a priori de Toledo, propuesto por LeSage y Krivelyova (1999) y LeSage y Pan (1995), y el enfoque de Minnesota. El BVAR permitirá incorporar la simultaneidad entre la criminalidad y desempleo; y la cercanía entre los estados de la frontera norte del país, evitando el problema del sobreajuste y colinealidad.

\subsection{La información a priori de Minnesota y Toledo}

Doan, Litterman y Sims (1984), y Litterman (1986), propusieron un enfoque bayesiano cuya información a priori, conocida como Minnesota, conduce a modelar una serie de tiempo como una caminata aleatoria simple. Con este enfoque, la atención se centra en el parámetro autorregresivo de primer orden, erradicando los problemas de colinealidad de los VAR estándar y mejorando sustancialmente la capacidad de pronóstico (LeSage y Cashell, 2015). La información a priori para la media y su varianza asociada sigue un distribución normal con

$$
N\left(1, \sigma_{\alpha_{i}}^{2}\right) \text { y } \alpha_{j} \sim N\left(0, \sigma_{\alpha_{j}}^{2}\right)
$$

donde $\alpha_{i}=1$ es la información a priori para el coeficiente autorregresivo y $\alpha_{j}=0$ es la información a priori para otras variables y rezagos mayores en el término autorregresivo.

Sin embargo, Dowd y LeSage (1997) señalan que el enfoque de Minnesota es inadecuado para modelar economías regionales, debido a que alguna presencia de co-movimiento sería más similar a un proceso de dependencia espacial que temporal. Este señalamiento fue recogido posteriormente por LeSage y Krivelyova (1999), quienes propusieron un enfoque que considera la interacción espacial entre las unidades regionales. 
Al igual que ocurre con la información a priori de Minnesota, la especificación de la información a priori espacial se determina de forma experimental con base en la asignación de hiperparámetros. Siguiendo la propuesta de LeSage y Krivelyova (1999), la información a priori, para la media de los coeficientes asociados a los estados contiguos retardados en un periodo de tiempo, es igual a $1 / C$, en la que $C$ corresponde al número de estados contiguos para cada variable endógena dependiente $i$. Por su parte, la información a priori para la media de los coeficientes asociados a los estados no contiguos es cero, así como también para el coeficiente asociado al primer rezago de cada variable endógena dependiente. Esta forma de asignar la información a priori para la media de los coeficientes implica que los estados contiguos ejercen una influencia relevante en las variables dependientes debido a la proximidad espacial.

Por su parte, la definición de la información a priori para la varianza de los coeficientes es esencial para apegarse a las características de los datos. En este sentido, una varianza pequeña implica que se otorga poca incertidumbre para que el coeficiente sea cercano a la información a priori, sobre su media; similarmente, una varianza mayor implica que el coeficiente estimado puede diferir de la información a priori, provista para la media. En este sentido, la asignación de la información a priori para la varianza se apega a los siguientes criterios: las variables asociadas a los estados no contiguos son menos relevantes que las asociadas a los estados contiguos debido a que la influencia decrece con la distancia, y la importancia de los retardos en el tiempo decrece a medida que el retardo es mayor, es decir, la influencia de las variables disminuyen con el tiempo.

La asignación de la información a priori para la media y varianza se expresa a través de (1), conforme a la propuesta de LeSage y Krivelyova (1999):

$$
\begin{gathered}
\pi\left(\beta_{i j k}\right)=N\left(1 / c_{i}, \sigma_{c}\right), j \in C, \text { para el rezago } k=1 ; i, j=1, \ldots, n, m . \\
\pi\left(\beta_{i j k}\right)=N\left(0, \tau \sigma_{c} / k\right), j \in C, \text { para el rezago } k=2 ; i, j=1, \ldots, n, m \\
\pi\left(\beta_{i j k}\right)=N\left(0, \theta \sigma_{c} / k\right), j \notin C, \text { para el rezago } k=1 ; i, j=1, \ldots, n, m
\end{gathered}
$$

Los hiperparámetros para la desviación estándar se definen como $0<\sigma_{c}<1$, y para el resto $\tau>1$ y $0<\theta<1$. En este sentido, para todas las variables $j$ en la ecuación $i$, contiguas al estado $i j \in C$, la información a priori para la media de los coeficientes en el primer rezago $(k=1)$ es el promedio del número de entidades contiguas a $i$, en tanto es cero para los estados no contiguos $j \notin C$. El valor a priori de la desviación estándar es igual a $\sigma_{c}$, para el primer rezago $(k=1)$, implicando una menor incertidumbre acerca del valor a priori asignado a la media de los coeficientes en el primer rezago, y por tanto, enfatizando la influencia de los estados contiguos. Por su parte, la 
información a priori para la varianza $\tau \sigma_{c} / k$, a partir del rezago $2(k=2)$, implica que la varianza disminuye conforme aumenta el número de rezagos $k$ en cada ecuación $i$; sin embargo, debido a que $\tau>1$, la incertidumbre se reduce de manera más suave permitiendo que el valor medio de cero, asignado a priori para los estados contiguos en los rezagos mayores a 1 , pueda diferir con el estimado ${ }^{3}$. La varianza $\theta \sigma_{c} / k$ asignada a priori impone de manera más estricta la media a priori de cero para los estados no contiguos, desde $k=1$ en adelante.

Recientemente, LeSage y Cashell (2015) señalan que el modelaje de las relaciones económicas regionales con base en un VAR que considere únicamente la dependencia espacial o temporal, sería inadecuado. Los autores reconocen la importancia de incorporar ambos tipos de dependencia, dando lugar a su propuesta de un enfoque híbrido que combine la información a priori de Minnesota y de Toledo.

\subsection{El modelo BVAR para analizar la relación entre desempleo y criminalidad}

La combinación de los enfoques de Minnesota y Toledo, permite elaborar un modelo BVAR, para estudiar la relación entre desempleo y criminalidad en los estados de la frontera norte de México, que considera endógenas a ambas variables. Este enfoque, denominado híbrido, permite incorporar efectos de dependencia temporal pero también espacial. En particular, el modelo en la expresión (2) establece que $d_{i t}$ y $r_{i t}$ representan la tasa de desempleo y la actividad delictiva en los estados de la frontera norte en el tiempo, medidas en tasas de crecimiento anualizadas, donde cada ecuación $i$ representa un estado en esta región para cada una de las variables $d_{i t}$ y $r_{i t}$. La expresión $D_{i j}=\sum_{j=1}^{m} D_{i j}$ define una matriz de pesos normalizada a 1 por renglones, que refleja la creencia a priori basada en el enfoque híbrido (ver cuadro A1 en anexos). En este sentido, cada variable endógena depende de variables explicativas, que son un promedio sobre las observaciones del estado $i$ y sus vecinos inmediatos con un rezago en el tiempo, aspecto que preserva la naturaleza parsimoniosa del promediado bayesiano ${ }^{4}$.

\footnotetext{
${ }^{3}$ La asignación de una varianza más suave que decrece conforme aumenta el número de rezagos es compatible con la idea de que la influencia de los estados contiguos en la variable dependiente i puede ser diferente de cero. Una forma de permitir que los datos dominen la información a priori para la media, es otorgando una relativa mayor incertidumbre, es decir, una varianza relativamente mayor.

${ }^{4}$ Desde luego, aunque el interés no consiste en evaluar las magnitudes de los coeficientes sino las funciones de impulso-respuesta derivadas, debe mencionarse que los coeficientes
} 


$$
\left[\begin{array}{l}
d_{i t} \\
r_{i t}
\end{array}\right]=\left[\begin{array}{ll}
D_{i j}(\ell) & D_{i j}(\ell) \\
D_{i j}(\ell) & D_{i j}(\ell)
\end{array}\right]\left[\begin{array}{c}
d_{j t-1} \\
r_{j t-1}
\end{array}\right]+\left[\begin{array}{l}
C_{d i t} \\
C_{r i t}
\end{array}\right]+\left[\begin{array}{c}
\varepsilon_{d i t} \\
\varepsilon_{r i t}
\end{array}\right]
$$

\subsection{Bases de datos y descripción de variables}

Las variables que se utilizaron en el BVAR corresponden a la tasa de desocupación abierta mensual que publica el Instituto Nacional de Estadística, Geografía e Informática (INEGI), con frecuencia mensual, para el periodo de marzo de 2005 a octubre de 2013, en los estados de la frontera norte: Baja California, Sonora, Chihuahua, Coahuila, Nuevo León y Tamaulipas. Por su parte, la tasa de criminalidad corresponde al número total de robos también para cada uno de los estados de la frontera norte del país. La información sobre el número de robos se obtuvo del Secretariado Ejecutivo del Sistema Nacional de Seguridad Pública (SESNSP), a partir de las estadísticas de incidencia delictiva por entidad federativa ${ }^{5}$. Debido a que el valor de la variable dependiente endógena, es igual al promedio de los valores en las variables de los estados contiguos, se requiere transformar las variables para que los valores tengan magnitudes similares. En este sentido, las variables fueron transformadas en tasas de crecimiento anualizadas.

De conformidad con lo descrito en la sección 3.1, se asignó un valor a priori de 0.5 a los coeficientes de la media, para cada variable dependiente autorregresiva con retardo de un periodo; y un valor a priori de $0.5\left(1 / c_{i}\right)$, para el primer retardo de los coeficientes de la media asociados a los estados contiguos (ver cuadro A1 en anexos). El valor asignado a los del hiperparámetro, para la desviación estándar de los coeficientes en el primer retardo, es $=0.2$; para la varianza de los coeficientes de los estados vecinos, en dos o más rezagos, es $=8$; y, para la varianza de los coeficientes para los estados no contiguos, en el primero o más rezagos, es $=1$. El número óptimo de rezagos en el VAR, se calculó con base en la prueba de la razón de verosimilitud. Con una significación estadística del uno

estimados dependen de los datos y del valor asignado a los hiperparámetros, por lo que el hecho de representar la matriz como $\mathrm{D}$, no implica que serán iguales.

${ }^{5}$ Es importante señalar que el Secretariado Ejecutivo del Sistema Nacional de Seguridad Pública, reporta la incidencia delictiva registrada en las averiguaciones previas o carpetas de investigación iniciadas por las Procuradurías Generales de Justicia y Fiscalías Generales de las entidades federativas, y por la Procuraduría General de la República. Sin embargo, debido a que no todos los delitos se reportan por diversos motivos o, incluso, no llegan a presumirse como delitos por falta de información, la cifra negra del delito estimada a partir de la ENVIPE levantada por el INEGI es elevada, siendo mayor de 90 por ciento en algunos años. Esta situación conduce a una subestimación del delito y, por tanto, es posible que incida en las magnitudes y trayectorias estimadas. 
por ciento, el rezago óptimo que se obtuvo es de 2 . Asimismo, las variables se definieron de la siguiente manera: dbc, dson, dchi, dcoa, dnl y dtam representan la tasa de desempleo para Baja California, Sonora, Chihuahua, Coahuila, Nuevo León y Tamaulipas, respectivamente. Similarmente, rbc, rson, rchih, rcoa, rnl y rtam se refieren al número de delitos totales para los estados fronterizos.

\section{Evidencia empírica}

En la gráfica 2, se aprecia la respuesta acumulativa que la tasa de desempleo y el número de robos tendría ante un choque sorpresivo que aumente la tasa de desempleo en el estado de Baja California, en una desviación estándar. El resultado muestra que la tasa de desempleo del estado de Sonora, contiguo inmediato a Baja California, tendría un ligero incremento inicialmente para que después de estabilizarse en dicho nivel, el efecto tendería a desaparecer aproximadamente un año más tarde. También se aprecia que la tasa de desempleo en Chihuahua recibiría el impacto de forma indirecta, aunque en el modelo se asignó a priori un coeficiente igual a cero, lo cual parece evidenciar que los datos estarían revelando la interdependencia económica entre los tres estados. En este caso, la tasa de desempleo de Chihuahua estaría respondiendo a efectos que provienen de la interdependencia con Baja California o, de forma secundaria, vía Sonora. De modo similar, el efecto se desvanecería a partir del primer año.

Con respecto a la respuesta en el número de robos, se observa que esta actividad delictiva respondería de forma retardada en Baja California, ya que a partir del segundo mes se observaría un aumento, sin embargo, sería a partir del séptimo mes cuando el número de robos disminuiría, ante la también disminución en la tasa de desempleo en Baja California. No obstante, en el mediano plazo, se esperaría que el número de robos se estabilice en un nivel mayor al inicial, cuando ocurrió el choque. El efecto también parece incidir en el número de robos en Sonora, el cual reaccionaría más rápidamente que Baja California incluso, para responder hacia la baja ante la caída acumulada en la tasa de desempleo. En particular, el número de robos en Sonora se mantendría fluctuante alrededor de su nivel inicial ante un posible aumento inicial en la tasa de desempleo de Baja California. Llama la atención que el número de robos en Chihuahua parece responder, también indirectamente, al aumento en la tasa de desempleo de Baja California, respondiendo de forma retardada durante el proceso en el que la tasa de desempleo comienza a disminuir. En ambos casos, el efecto se desvanece prácticamente a los dos años (gráfica 2). 
Gráfica 2

Respuesta acumulada de la tasa de desempleo y el número de robos en los estados de la frontera norte ante un choque de una desviación estándar a la ecuación de la tasa de desempleo en Baja California (dbc)

Tasa de desempleo
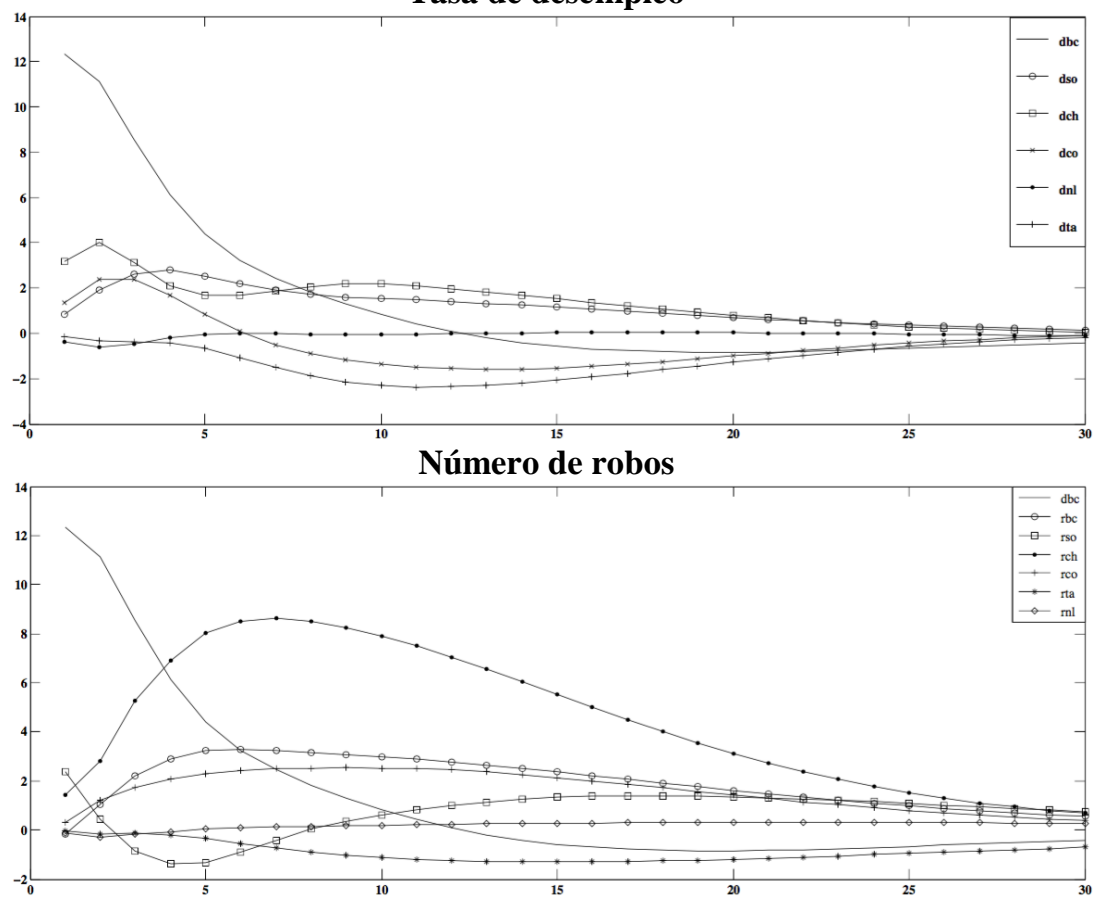

Fuente: estimaciones propias.

En la gráfica 3, se observa la respuesta de la tasa de desempleo y el número de robos ante un aumento sorpresivo en la tasa de desempleo de Sonora, también de una desviación estándar. En este caso, si bien los estados vecinos de Baja California y Chihuahua parecen no responder contemporáneamente de forma inmediata, con excepción de una caída en el segundo mes en la tasa de desempleo de Chihuahua, el comportamiento de la respuesta es bastante similar en ambos estados a partir del tercer mes. Una vez que la tasa de desempleo de Sonora comience a disminuir, el descenso en Baja California y Chihuahua comenzaría a partir del séptimo mes. Aunque el estado de Nuevo León se encuentra a una distancia mayor, se observa que pudiera recibir un efecto indirecto, aunque parece absorberlo rápidamente, ya que se estabiliza. 
Gráfica 3

Respuesta acumulada de la tasa de desempleo y el número de robos en los estados de la frontera norte ante un choque de una desviación estándar a la ecuación de la tasa de desempleo en Sonora (dso)

Tasa de desempleo
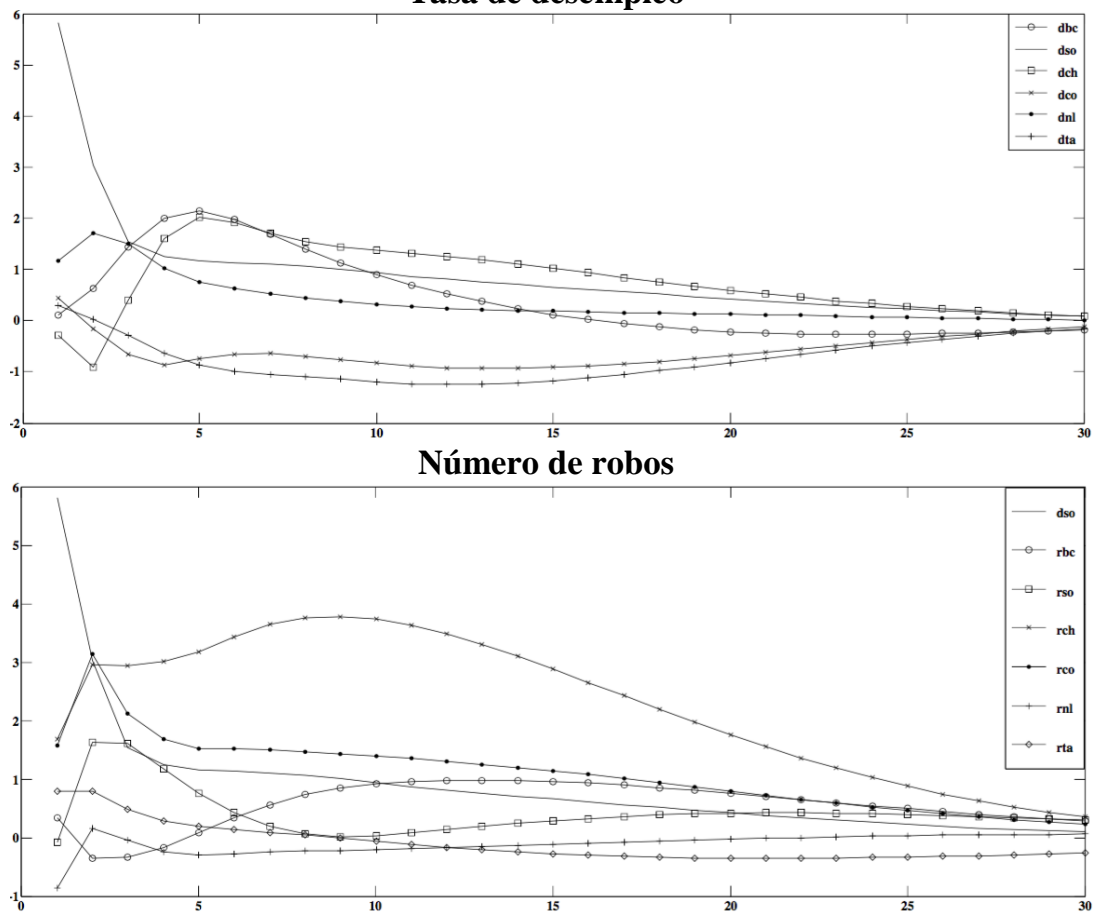

Fuente: estimaciones propias.

Un choque sorpresivo que eleve la tasa de desempleo del estado de Chihuahua se transmitiría de forma inmediata al estado de Baja California: la tasa de desempleo aumentaría en el primer mes, para empezar a disminuir a partir del segundo mes a la par que la tasa de desempleo en Chihuahua, aunque a un ritmo menor (gráfica 4). Por su parte, la tasa de desempleo en los estados de Sonora y Coahuila tendría una respuesta menor que la tasa de desempleo de Baja California, aunque ambos son vecinos contiguos de Chihuahua. En el caso de Coahuila, el desempleo aumentaría los primeros tres meses para empezar a disminuir a partir del cuarto mes. A diferencia de Sonora, cuya tasa de desempleo no aumentaría sino hasta los primeros cinco meses, para estabilizarse en un nivel más alto de desempleo en el mes quince. Esta trayectoria significa que el estado encontraría relativamente más complicado regresar a los niveles anteriores de desempleo, frente a un desajuste que eleve la tasa de desempleo en Chihuahua (gráfica 4). 
Por otro lado, el número de robos aumentaría significativamente en Chihuahua como resultado de un choque que también aumente su tasa de desempleo (gráfica 4). En este caso, el número de robos tendría una trayectoria ascendente hasta el noveno mes, sin embargo, tendrían que pasar aproximadamente veinticuatro meses para que el efecto se desvanezca. En el caso de Sonora, el número de robos disminuiría hasta el quinto mes, para posteriormente tomar una trayectoria ascendente y empezar a desvanecerse en el mes veinte. Baja California y Coahuila también experimentarían una trayectoria ascendente en el número de robos hasta los primeros doce meses, para iniciar posteriormente un descenso en esta actividad delictiva. Ambos estados tendrían la misma trayectoria a partir del mes doce, y Sonora se acoplaría hasta el mes veinte. El choque parece no transmitirse al número de robos que ocurren en los estados de Tamaulipas y Nuevo León.

\section{Gráfica 4}

Respuesta acumulada de la tasa de desempleo y el número de robos en los estados de la frontera norte ante un choque de una desviación estándar a la ecuación de la tasa de desempleo en Chihuahua (dch)

Tasa de desempleo

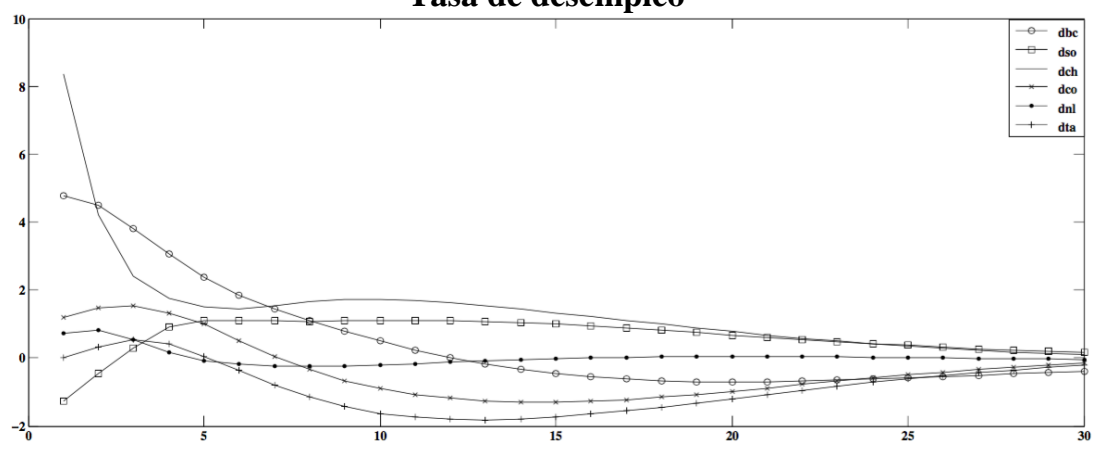

Número de robos

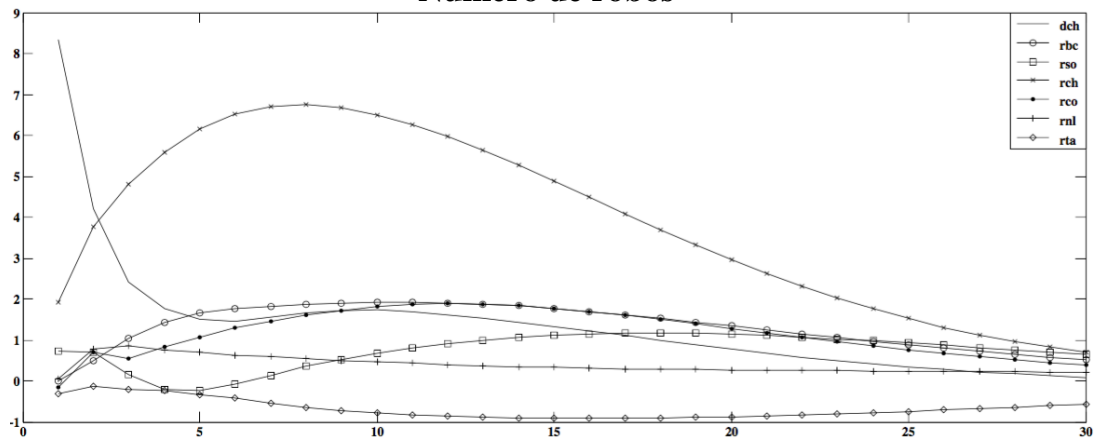

Fuente: estimaciones propias. 
Un aumento en la tasa de desempleo en el estado de Coahuila tendría efectos iniciales diferenciados en algunos estados. Por ejemplo, la tasa de desempleo en Baja California aumentaría inicialmente hasta los primeros cinco meses, para posteriormente disminuir hasta el mes quince, cuando parece recobrar su nivel inicial de desempleo. Sonora inicialmente experimentaría una disminución en la tasa de desempleo, aunque a partir del quinto mes iniciaría una trayectoria ascendente para empezar a disminuir nuevamente hasta el mes dieciséis. Sin embargo, la tasa de desempleo terminaría siendo ligeramente mayor que su nivel inicial, cuando Coahuila recibió el choque. Chihuahua también tendría un aumento; sin embargo, prácticamente en el sexto mes estaría recobrando sus niveles iniciales de desempleo. El estado de Tamaulipas parece beneficiarse del choque en Coahuila, ya que tendría una disminución temporal en su tasa de desempleo durante aproximadamente veinticinco meses (gráfica 5).

Aunque inicialmente el número de robos en Coahuila parecen responder inversamente ante un aumento en su tasa de desempleo, a partir del tercer mes iniciaría una trayectoria ascendente cuyo efecto empezaría a disminuir año y medio después, sin embargo, el número de robos tendería a ser mayor que cuando ocurrió el choque inicial a la tasa de desempleo. Llama la atención que el número de robos en Chihuahua aumentaría significativamente, aunque en menor magnitud que cuando se simuló el choque en su propia tasa de desempleo, pero con una trayectoria similar. Sin embargo, este impacto implica que Chihuahua enfrentaría un brote en el número de robos con duración de poco más de dos años, por un desajuste en el mercado laboral en el estado vecino. Con respecto a Baja California y Sonora, seguirían una trayectoria similar a la que experimentaría Coahuila, con un aumento en el número de robos apenas mayor al que tenían cuando ocurrió el choque (gráfica 5).

Por su parte, un choque que induzca un aumento en la tasa de desempleo del estado de Nuevo León, tendría un efecto incremental inicial importante en las tasas de desempleo de Baja California, Coahuila y Chihuahua y, en menor medida, en Tamaulipas (gráfica 6). No obstante, a medida que el efecto disminuye en Nuevo León, los estados de Baja California, Coahuila y Chihuahua también experimentan un descenso en la tasa de desempleo, aunque con menor rapidez; de hecho, la trayectoria indica que el nivel de desempleo terminaría siendo menor al inicial en estos tres estados, que cuando Nuevo León recibió el choque en su mercado laboral. Tamaulipas parece seguir una trayectoria descendente para terminar en niveles de desempleo similares a su tasa inicial. El estado de Sonora parece responder en menor medida, aunque también terminaría con un nivel de desempleo relativamente mayor a su nivel inicial, siendo este el estado que finalmente 
terminaría siendo más afectado ante un aumento súbito en la tasa de desempleo de Nuevo León (gráfica 6).

\section{Gráfica 5}

Respuesta acumulada de la tasa de desempleo y el número de robos en los estados de la frontera norte ante un choque de una desviación estándar a la ecuación de la tasa de desempleo en Coahuila (dco) Tasa de desempleo

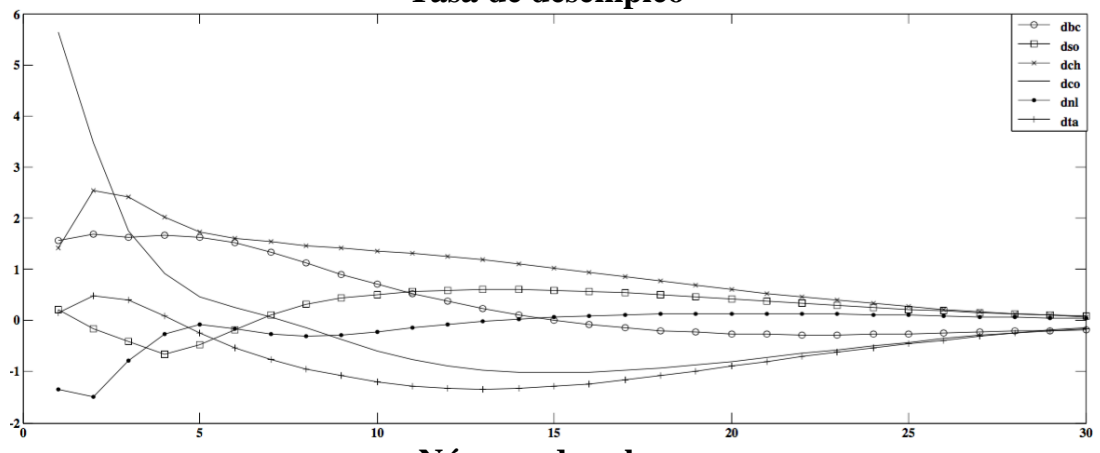

Número de robos

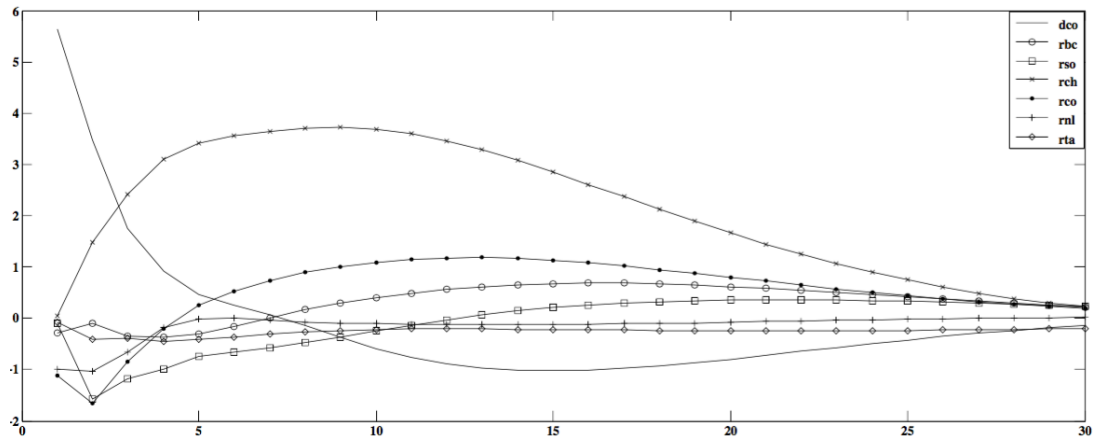

Fuente: estimaciones propias.

Con respecto al impacto que el aumento en la tasa de desempleo tendría en el número de robos, llama la atención que esta actividad delictiva parece responder poco ante desajustes en el mercado laboral, en el propio estado de Nuevo León; incluso, el efecto final dejaría intacto el número de robos. Igual que en Tamaulipas, el cual siendo vecino de Nuevo León, parece no recibir un impacto significativo. Sin embargo, esto no sería así en el resto de los estados fronterizos; por ejemplo, Baja California, Sonora y Coahuila, y principalmente el segundo, seguirían una trayectoria que les dejaría en niveles delictivos ligeramente mayores a los de sus niveles iniciales, respectivos. En el caso de Chihuahua, el impacto parece transmitirse nuevamente de forma significativa, sin embargo, aunque esta entidad parece ajustarse con dificultad al atravesar por periodos de intensificación en el 
número de robos, al final tendería a permanecer en niveles similares que al principio del choque (gráfica 6).

En este sentido, los efectos en este tipo de delito adquisitivo parecen complicar la inseguridad en los estados fronterizos en diferentes formas: mientras se somete a un periodo de intensificación temporal en Chihuahua, para regresar a niveles similares al inicial; el impacto parece ocasionar un aumento estructural de este delito en Baja California, Sonora y Coahuila, ya que el número de robos convergería en un nivel mayor al inicial.

\section{Gráfica 6}

Respuesta acumulada de la tasa de desempleo y el número de robos en los estados de la frontera norte ante un choque de una desviación estándar a la ecuación de la tasa de desempleo en Nuevo León (dnl)
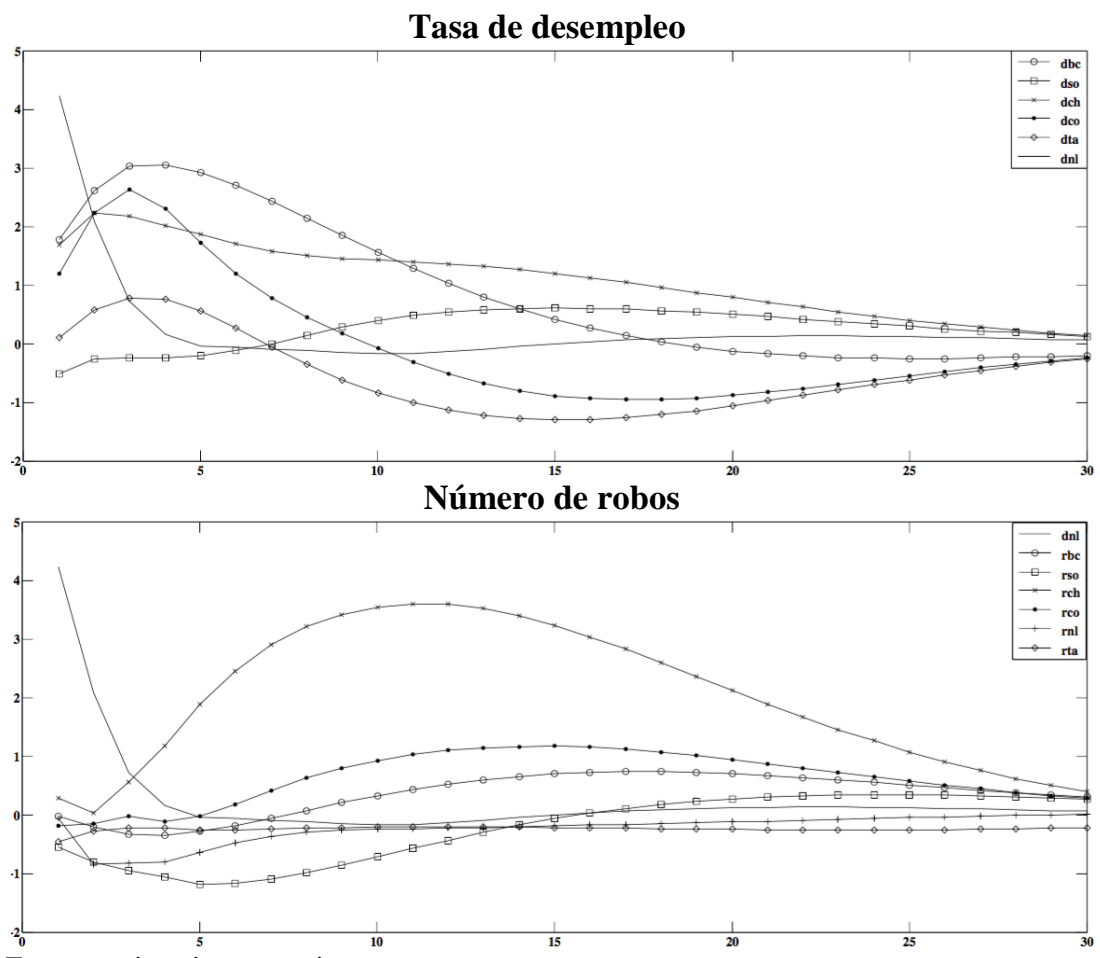

Fuente: estimaciones propias.

Cuando se evalúa el impacto que un aumento en la tasa de desempleo del estado de Tamaulipas tendría sobre los estados de la frontera norte, se observa que la tasa de desempleo respondería ligeramente a la alza en el primer mes, con excepción de Nuevo León, que inicialmente disminuiría. No 
obstante, Nuevo León tendría un trayectoria ascendente durante el primer año, que conduciría a un nivel de desempleo ligeramente mayor que su nivel inicial. El resto de los estados tendrían una trayectoria que los llevaría a niveles iguales o menores de desempleo. De hecho, el efecto acumulativo es compatible con la poca respuesta que la tasa de desempleo de Tamaulipas observa ante choques en otros estados, lo que parece indicar una menor integración económica entre Tamaulipas y el resto de estados fronterizos, por lo menos con respecto a la interrelación entre sus mercados laborales (gráfica 7).

\section{Gráfica 7}

Respuesta acumulada de la tasa de desempleo y el número de robos en los estados de la frontera norte ante un choque de una desviación estándar a la ecuación de la tasa de desempleo en Tamaulipas (dta)

Tasa de desempleo

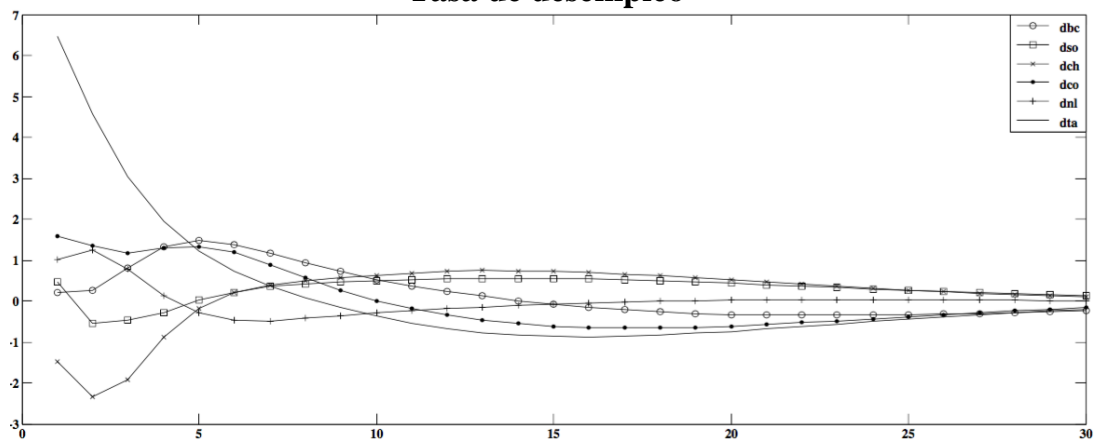

Número de robos

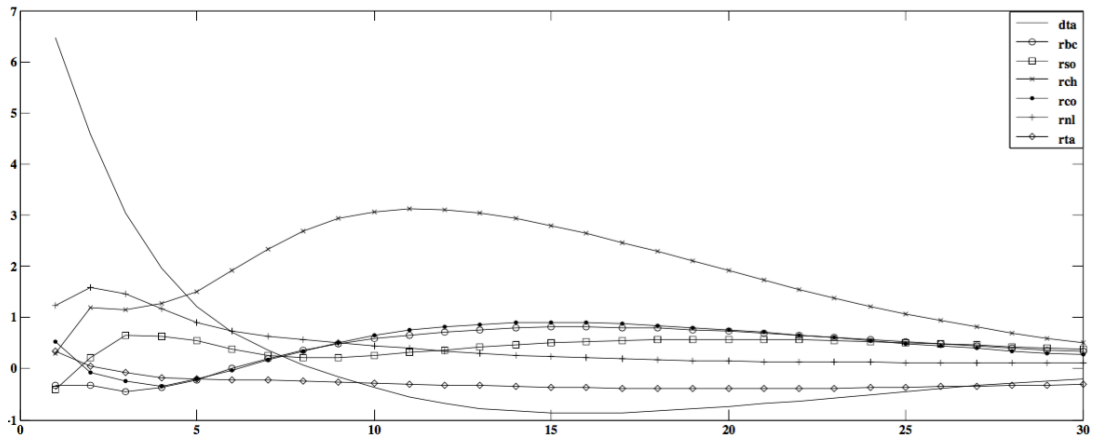

Fuente: estimaciones propias.

Similarmente, el efecto en el número de robos parece transmitirse en menor medida cuando se compara con el resto de gráficas. Mientras el número de robos en Tamaulipas parece no responder ante un aumento en su tasa de desempleo; en Nuevo León, tendería a desvanecerse en los primeros cuatro meses (gráfica 7). El resto de los estados responderían con una trayectoria 
similar a la experimentada ante los choques en las gráficas anteriores; por ejemplo, Chihuahua tendría un aumento temporal comparativamente mayor que el resto de estados en el número de robos, para finalizar en un nivel similar al inicial; en tanto, Baja California y Sonora tendrían una trayectoria ascendente, aunque menor a Chihuahua, que en su caso los conduciría a un nivel de actividad delictiva ligeramente mayor. Y el estado de Coahuila convergería con sus niveles iniciales de delitos por robos (gráfica 7).

\section{Conclusiones}

En esta investigación, se analizó la relación entre actividad criminal y la tasa de desempleo en los estados ubicados en la frontera norte de México. En particular, se evalúa el impacto acumulativo que un choque sorpresivo que aumente la tasa de desempleo en los diferentes estados de la frontera norte, tendría en la tasa de desempleo y el número de robos en esta región. Aunque la relación tasa de desempleo-actividad delictiva ha sido abordada en diferentes estudios empíricos internacionales, en el caso de México aún es una relación que requiere mayor investigación. Su importancia estriba en que las fluctuaciones de la actividad económica que influyen en la tasa de desempleo están vinculadas con la comisión de delitos, particularmente aquellos de tipo adquisitivo como los robos, convirtiéndose entonces en un estudio relevante para que quienes elaboran e implementan las medidas de seguridad pública, consideren la evolución del mercado laboral.

El análisis contribuye al conocimiento de la relación empírica entre criminalidad y desempleo para esta región de México, aunque es insuficiente para conducir a algún hecho estilizado y generalizado para el resto del país. En este sentido, la evidencia empírica sugiere que la respuesta del número de robos a un choque que aumente la tasa de desempleo es diferenciada entre los estados de la frontera norte. En particular, se observa que los estados presentan una relación positiva de forma consistente ante un choque que incida en la tasa de desempleo en cualquier estado de la frontera norte. La relación parece reflejar un efecto de motivación económica, en la que incrementos (disminuciones) en la tasa de desempleo se asocian con incrementos (disminuciones) en la actividad delictiva.

La trayectoria que sigue el número de robos en Chihuahua es peculiar, ya que prácticamente un incremento en el desempleo de cualquier estado fronterizo parece promover un aumento en los robos de dicho estado, aunque su efecto sea temporal en el mediano plazo. En cambio, en algunos estados como Baja California, Sonora y Coahuila, el número de robos parece corresponder en menor medida, con lo que sucede en Chihuahua; sin embargo, estos tres estados terminarían convergiendo en un nivel delictivo ligeramente mayor al 
inicial. Llama la atención que estas tres entidades parecen reflejar una importante interacción espacial, ya que los choques que ocurren en algunos de estos estados se traducen en trayectorias similares en la actividad delictiva, pero diferentes con el resto de estados fronterizos.

En el estado de Tamaulipas, el número de robos parece reaccionar con menor sensibilidad ante choques que eleven la tasa de desempleo de este estado u otro en la frontera norte. Lo que también refleja posiblemente una menor interacción espacial. Esta relación diferenciada entre actividad delictiva de tipo adquisitivo y desempleo en los estados de la frontera norte, explicada en parte por la asociación espacial considerada en el análisis, tiene implicaciones importantes para la política económica y de seguridad pública.

En este sentido, los resultados sugieren que ambas políticas deben estar sincronizadas debido a que, por ejemplo, las políticas de disminución del desempleo estarían promoviendo en algunos estados una alza en la actividad delictiva, por lo menos en el corto plazo. Asimismo, dichas políticas deben tener un carácter regional considerando que la interacción espacial observada parece favorecer efectos de traspaso entre estados vecinos, que en algunos casos pueden ser favorables en términos económicos y de seguridad. Por esta razón, las políticas de cooperación regional que enfrenten los problemas de desempleo y seguridad pública son necesarias para alcanzar beneficios colectivos.

Por otro lado, la metodología empleada en el análisis de la relación actividad criminal-tasa de desempleo, además de contribuir a aliviar el problema del sobreajuste y considerar la interacción espacial entre los estados de la frontera norte, permite avanzar en la identificación de regularidades empíricas y proveer la base para un análisis económico más profundo. En este sentido, esta investigación puede extenderse para considerar la presencia de variables macroeconómicas que pueden influir a escala regional, un vínculo macro-regional poco abordado en la literatura internacional y nacional. Asimismo, este trabajo significa un primer paso para la elaboración de modelos de equilibrio general dinámicos estocásticos, que reproduzcan las relaciones regionales expuestas.

Finalmente, debe mencionarse que si bien las Naciones Unidas reconoce que la tasa de desempleo ha sido frecuentemente utilizada en estudios internacionales como medida de actividad económica para investigar su relación con la criminalidad (UNODC, 2011), es prudente plantear el alcance y limitaciones en el análisis. En primera instancia, la tasa de desempleo dista de ser un indicador perfecto de la actividad económica, ya que suele subrepresentar algunos factores, como el acceso al crédito, la reducción de gasto público social, entre otros. Adicionalmente, la actividad delictiva también 
puede estar asociada con otras dimensiones sociales, por ejemplo, las Naciones Unidas identifican ocho subsistemas que inciden en la comisión del delito: demográficos, familiares, laborales (desempleo), económicos (pobreza, desigualdad, entre otros), políticos, institucionales, culturales asociados al consumo de alcohol, drogas y porte de armas, y culturales asociados a la tolerancia o legitimación de la violencia o la trampa (PNUD, 2009).

\section{Referencias}

[1] Becker, G. (1968). "Crime and punishment: an economic approach". The Journal of Political Economy, 76 (2), 169-217.

[2] Bonger, W. (1916). Criminality and economic conditions. Primera edición, Boston, USA: Little, Brown and Company.

[3] Britt, C. (1997). "Reconsidering the unemployment and crime relationship: variations by age group and historical period". Journal of Quantitative Criminology, 13 (4), 405-428.

[4] Cantor, D. y Land, K. (1985). "Unemployment and crime rates in the Post WorldWar II United States: a theoretical and empirical analysis". American Sociological Review, 50 (3), 317-332.

[5] Ciccarelli, M. y Rebucci, R. (2003), "Bayesian VARS: A survey of the recent literature with an application to the European Monetary System". International Monetary Fund Working Paper WP/03/102.

[6] Chiricos, T. (1987). "Rates of crime and unemployment: an analysis of aggregate research evidence". Social Problems, 34 (2), 187-212.

[7] Cook, S. y Watson, D. (2014). "A re-examination of the opportunity and motivation effects underlying criminal activity". Criminology and criminal justice, 14 (4), 458-469.

[8] Cornwell, C. y Trumbull, W. (1994). "Estimating the economic model of crime with panel data". The Review of Economics and Statistics, 76 (2), 360-366.

[9] Daniele, V. y Marani, U. (2010). "Organized crime and foreign direct investment: the Italian case". CESIFO Woking Paper No. 2416.

[10] Detotto, C. y Otranto, E. (2012). "Cycles in crime and economy: leading, lagging and coincident behaviors". Journal of Quantitative Criminology, 28 (2), 295-317.

[11] Doan, T., Litterman, r. y Sims, C. (1984). "Forecasting and conditional projections using realistic prior distributions". Econometric Reviews 3 (1), $1-100$.

[12] Dowd, M. and LeSage, J. (1997). "Analysis of spatial contiguity influences on state price level formation". International Journal of Forecasting, 13 (2), 245-253.

[13] Ehrlich, I. (1967). "The supply of illegitimate activities". Manuscrito sin publicar. Columbia University.

[14] Ehrlich, I. (1973). "Participation in illegitimate activities: a theoretical and empirical investigation". Journal of Political Economy, 81(3), 521-565. 
[15] Enamorado, T., López, L. y Rodríguez, C. (2013). "Crime and growth convergence, evidence from Mexico". The World Bank Policy Research Working Paper No. 6730.

[16] Feliz, R. (2012). Crimen y crecimiento económico en México. Los estados federales en el periodo 2003-2010. En Aguilar, J. (Coordinador), Las bases sociales del crimen organizado y la violencia en México (220-245). México: CIES y SSP.

[17] Fleisher, B. (1966). The Economics of delinquency. Primera Edición, USA: Quadrangle.

[18] González, S (2014). "Criminalidad y crecimiento económico regional en México". Frontera Norte, 26 (51), 75-111.

[19] Goulas, E. y Zervoyianni, A. (2013). "Economic growth and crime: does uncertainty matter?". Applied Economic Letters, 20 (5), 420-427.

[20] Krkoska, L. y Robeck, K. (2009). "Crime, business conduct and investment decisions: Enterprise survey evidence from 34 countries in Europe and Asia". Review of Law and Economics, 89 (4), 601-614.

[21] Kumar, S. (2013). "Crime and economic growth: evidence from India". MPRA Working Paper No. 48794.

[22] LeSage, J. y Pan, Z. (1995). "Using spatial contiguity as bayesian prior information in regional forescating models". International Regional Science Review, 18 (1), 33-53.

[23] LeSage, J. y Krivelyova, A. (1999). "A spatial prior for bayesian vector autoregressive models". Journal of Regional Science, 39 (2), 297-317.

[24] LeSage, J. Y Bryce, A. Cashell (2015). “A comparison of vector autoregressive forecasting performance: spatial versus non-spatial Bayesian priors". The Annals of Regional Science, 54 (2), 533-560.

[25] Levitt, S. (2001). "Alternative strategies for indentifying the link between unemployment and crime". Journal of Quantitative Criminology, 17 (4), 377-390.

[26] Litterman, R. (1986). "Forecasting with Bayesian Vector Autoregressions: five years of experience". Journal of Business and Economic Statistics, 4 (1), 25-38.

[27] Mendoza, E. (2013). Introducción. En Mendoza, E. (Coordinador), La crisis financiera internacional: efectos sectoriales en México y en su frontera norte (11-27). México: El Colef.

[28] Myers, S. (1983). "Estimating the economic model of crime: employment versus punishment effects". Quarterly Journal of Economics, 98 (1), 157-166.

[29] Neanidis, K. and Papadoupolou, V. (2013). "Crime, fertility and economic growth: Theory and evidence". Journal of Economic Behavior and Organization, 91 (July), 101-121.

[30] Öcal N. y Yildirim, J. (2010). "Regional effects of terrorism on economic growth in Turkey: a geographically weighted regression approach". Journal of Peace Research, 47 (4), 477-489.

[31] Osorio, J. (2012). Las causas estructurales de la violencia: evaluación de algunas hipótesis. En Aguilar, J. (Coordinador), Las bases sociales del crimen organizado y la violencia en México (72-130). México: CIES y SSP.

[32] Pan, M., Widner, B. y Enomoto, C. (2012). "Growth and crime in contiguous states of Mexico". Review of Urban \& Regional Development Studies, 24 (1-2), 51-64. 
[33] Phelps, H. (1929). "Cycles in crime". Journal of the American Institute of Criminal Law and Criminology, 20 (1), 107-121.

[34] Phillips, J. y Land, K. (2012). "The link between unemployment and crime rate fluctuations: an analysis at the county, state, and national levels", Social Science Research, 41 (3), 681-694.

[35] PNUD (2009). "Informe sobre el desarrollo humano para América Central, 20092010: abrir espacios para la seguridad ciudadana y el desarrollo humano". Programa de las Naciones Unidas para el Desarrollo. Colombia: PNUD.

[36] Schmitd, P. y Witte, A. (1984). Estimating a simple economic model of criminal behavior. En Schmitd, P., A. Witte y P. Rossi (Eds.), An economic analysis of crime and justice: theory, methods and applications (194-216). USA: Academic Press.

[37] Smith, D., Devine, J. y Sheley, J. (1992). "Crime and unemployment: effects across race and age categories". Sociological perspectives, 35 (4), 551572.

[38] Smigel-Leibowitz, A. (1965). Does crime pay? An Economic Analysis. Tesis inédita de Maestría. Columbia University.

[39] SBP (2008). "The impact of crime on the small businesses in South Africa". Small Business Project. South Africa: Small Business Project.

[40] Torres Preciado, V.H. (2013). La crisis económico-financiera y su efecto en la tasa de desempleo en Baja California: un análisis VAR contrafactual y de descomposición histórica. En Mendoza, E. (Coordinador), La crisis financiera internacional: efectos sectoriales en México y en su frontera norte (255-284 ). México: El Colef.

[41] Torres Preciado, V.H. y Polanco, M. (2014). Ciclo económico y criminalidad en los estados de la región centro occidente de México: implicaciones para la estrategia nacional de seguridad pública. En Mejía, P. y Torres Preciado, V. H. (Coordinadores), Efectos de las reformas estructurales en las fluctuaciones cíclicas y el crecimiento económico en México (95-114). México: UAEM y Eón.

[42] Torres Preciado, V.H., Polanco, M. y Venegas, F. (2015). “Actividad criminal e inversión privada en México: una perspectiva espacial, 1997-2010". Papeles de Población, 21 (83), 203-251.

[43] Torres Preciado, V.H., Polanco, M. y Tinoco, M. (2015). "Crime and regional economic growth in Mexico: a spatial perspective". Papers in Regional Science. DOI: 10.1111/pirs. 12205

[44] Thomas, D. (1925). Social aspects of the business cycles. Primera edición, Nueva York, USA: Routledge and Sons.

[45] UNODC (2011). "Monitoring the impact of economic crisis on crime". United Nations Office on Drugs and Crime. Austria: United Nations Office on Drugs and Crime.

[46] Verdugo-Yepes, C., Pedroni, P. y Hu, X. (2015). "Crime and the Economy in the Mexican states: heterogeneous panel estimates (1993-2012). IMF Working Paper No. 121.

[47] Witte, A. (1980). "Estimating the economic model of crime with individual data". Quarterly Journal of Economics, 94 (1), 57-84.

[48] Yamada, T. (1985). "The crime rate and the condition of the labor market: a vector autoregressive model". National Bureau of Economic Research Working Paper No. 1782. 
[49] Yamada, T., Yamada, T. y Kang, J. (1991). "Crime rates versus labor market conditions; theory and time-series evidence". National Bureau of Economic Research No. 3801.

[50] Zelekha, Y. y Bar-Efrat, O. (2011). "Crime, terror and corruption and their effect on private investment on Israel". Open Economies Review, 22 (4), 635648.

\section{Anexos}

Cuadro A1

Matriz de pesos con la información a priori híbrida para la media de los coeficientes

\begin{tabular}{|c|c|c|c|c|c|c|c|c|c|c|c|c|}
\hline Variable & $\mathrm{dbc}$ & dso & dch & dco & $\mathrm{dnl}$ & dta & $\mathrm{cbc}$ & cso & $\mathrm{cch}$ & cco & $\mathrm{cnl}$ & cta \\
\hline $\mathrm{dbc}$ & 0.250 & 0.250 & 0.000 & 0.000 & 0.000 & 0.000 & 0.250 & 0.250 & 0.000 & 0.000 & 0.000 & 0.000 \\
\hline dso & 0.125 & 0.250 & 0.125 & 0.000 & 0.000 & 0.000 & 0.125 & 0.250 & 0.125 & 0.000 & 0.000 & 0.000 \\
\hline dch & 0.000 & 0.125 & 0.250 & 0.125 & 0.000 & 0.000 & 0.000 & 0.125 & 0.250 & 0.125 & 0.000 & 0.000 \\
\hline dco & 0.000 & 0.000 & 0.125 & 0.250 & 0.125 & 0.000 & 0.000 & 0.000 & 0.125 & 0.250 & 0.125 & 0.000 \\
\hline $\mathrm{dnl}$ & 0.000 & 0.000 & 0.000 & 0.125 & 0.250 & 0.125 & 0.000 & 0.000 & 0.000 & 0.125 & 0.250 & 0.125 \\
\hline dta & 0.000 & 0.000 & 0.000 & 0.000 & 0.250 & 0.250 & 0.000 & 0.000 & 0.000 & 0.000 & 0.250 & 0.250 \\
\hline $\mathrm{cbc}$ & 0.250 & 0.250 & 0.000 & 0.000 & 0.000 & 0.000 & 0.250 & 0.250 & 0.000 & 0.000 & 0.000 & 0.000 \\
\hline cso & 0.125 & 0.250 & 0.125 & 0.000 & 0.000 & 0.000 & 0.125 & 0.250 & 0.125 & 0.000 & 0.000 & 0.000 \\
\hline cch & 0.000 & 0.125 & 0.250 & 0.125 & 0.000 & 0.000 & 0.000 & 0.125 & 0.250 & 0.125 & 0.000 & 0.000 \\
\hline cco & 0.000 & 0.000 & 0.125 & 0.250 & 0.125 & 0.000 & 0.000 & 0.000 & 0.125 & 0.250 & 0.125 & 0.000 \\
\hline $\mathrm{cnl}$ & 0.000 & 0.000 & 0.000 & 0.125 & 0.250 & 0.125 & 0.000 & 0.000 & 0.000 & 0.125 & 0.250 & 0.125 \\
\hline cta & 0.000 & 0.000 & 0.000 & 0.000 & 0.250 & 0.250 & 0.000 & 0.000 & 0.000 & 0.000 & 0.250 & 0.250 \\
\hline
\end{tabular}

Fuente: elaboración propia.

\section{Cuadro A2}

\section{Número de delitos totales en los estados de la frontera norte de México}

\begin{tabular}{llllllllll}
\hline Año & Nacional & $\begin{array}{l}\text { Frontera } \\
\text { norte }\end{array}$ & $\%$ & $\begin{array}{l}\text { Baja } \\
\text { California }\end{array}$ & Sonora & Chihuahua & Coahuila & $\begin{array}{l}\text { Nuevo } \\
\text { León }\end{array}$ & Tamaulipas \\
\hline 2005 & 1415681 & 334,064 & 23.6 & 101,166 & 43,512 & 67,690 & 24,927 & 44,254 & 52,515 \\
2006 & 1458580 & 343,554 & 23.6 & 112,081 & 37,321 & 67,766 & 26,928 & 46,212 & 53,246 \\
2007 & 1587030 & 359,765 & 22.7 & 129,212 & 40,489 & 51,825 & 32,891 & 53,554 & 51,794 \\
2008 & 1628054 & 390,005 & 24.0 & 133,762 & 35,776 & 69,275 & 44,115 & 51,863 & 55,214 \\
2009 & 1653260 & 370,917 & 22.4 & 123,014 & 30,506 & 67,815 & 49,798 & 46,489 & 53,295 \\
2010 & 1707441 & 359,575 & 21.1 & 110,811 & 29,410 & 69,632 & 49,777 & 52,312 & 47,633 \\
2011 & 1729934 & 365,630 & 21.1 & 115,328 & 30,769 & 65,560 & 48,882 & 56,230 & 48,861 \\
2012 & 1704915 & 339,216 & 19.9 & 108,682 & 34,198 & 61,039 & 48,642 & 42,346 & 44,309 \\
2013 & 1681077 & 330,039 & 19.6 & 104,029 & 34,492 & 61,478 & 49,329 & 43,972 & 36,739 \\
2014 & 1590331 & 333,225 & 21.0 & 107,284 & 31,182 & 58,674 & 51,412 & 40,036 & 44,637 \\
2015 & 1259014 & 271,466 & 21.6 & 87,875 & 23,922 & 52,201 & 39,866 & 31,732 & 35,870 \\
\hline
\end{tabular}

Fuente: elaboración propia con información del Secretariado Ejecutivo del Sistema Nacional de Seguridad Pública. Nota: la información para 2015 abarca hasta octubre. 
Cuadro A3

Distribución porcentual del número de delitos totales en los estados de la frontera norte de México

\begin{tabular}{|c|c|c|c|c|c|c|c|}
\hline Año & $\begin{array}{l}\text { Frontera } \\
\text { norte }\end{array}$ & $\begin{array}{c}\text { Baja } \\
\text { California } \\
\%\end{array}$ & Sonora $\%$ & $\begin{array}{c}\text { Chihuahua } \\
\%\end{array}$ & Coahuila \% & $\begin{array}{l}\text { Nuevo } \\
\text { León \% }\end{array}$ & $\begin{array}{c}\text { Tamaulipas } \\
\%\end{array}$ \\
\hline 2005 & 334,064 & 30.3 & 13.0 & 20.3 & 7.5 & 13.2 & 15.7 \\
\hline 2006 & 343,554 & 32.6 & 10.9 & 19.7 & 7.8 & 13.5 & 15.5 \\
\hline 2007 & 359,765 & 35.9 & 11.3 & 14.4 & 9.1 & 14.9 & 14.4 \\
\hline 2008 & 390,005 & 34.3 & 9.2 & 17.8 & 11.3 & 13.3 & 14.2 \\
\hline 2009 & 370,917 & 33.2 & 8.2 & 18.3 & 13.4 & 12.5 & 14.4 \\
\hline 2010 & 359,575 & 30.8 & 8.2 & 19.4 & 13.8 & 14.5 & 13.2 \\
\hline 2011 & 365,630 & 31.5 & 8.4 & 17.9 & 13.4 & 15.4 & 13.4 \\
\hline 2012 & 339,216 & 32.0 & 10.1 & 18.0 & 14.3 & 12.5 & 13.1 \\
\hline 2013 & 330,039 & 31.5 & 10.5 & 18.6 & 14.9 & 13.3 & 11.1 \\
\hline 2014 & 333,225 & 32.2 & 9.4 & 17.6 & 15.4 & 12.0 & 13.4 \\
\hline 2015 & 271,466 & 32.4 & 8.8 & 19.2 & 14.7 & 11.7 & 13.2 \\
\hline
\end{tabular}

Cuadro A4

Número de robos en los estados de la frontera norte de México

\begin{tabular}{llllllllll}
\hline Año & \multirow{2}{*}{ Nacional } & $\begin{array}{l}\text { Frontera } \\
\text { norte (FN) }\end{array}$ & $\begin{array}{l}\% \\
\text { FN }\end{array}$ & $\begin{array}{l}\text { Baja } \\
\text { California }\end{array}$ & Sonora & Chihuahua & Coahuila & $\begin{array}{l}\text { Nuevo } \\
\text { León }\end{array}$ & Tamaulipas \\
\hline 2005 & 1415681 & 149,694 & 10.6 & 51778 & 13126 & 31349 & 9412 & 23233 & 20796 \\
2006 & 1458580 & 165,792 & 11.4 & 59439 & 12487 & 35085 & 9916 & 26449 & 22416 \\
2007 & 1587030 & 187,013 & 11.8 & 72838 & 14349 & 29428 & 11662 & 35472 & 23264 \\
2008 & 1628054 & 203,260 & 12.5 & 75075 & 11,967 & 39146 & 17299 & 36279 & 23,494 \\
2009 & 1653260 & 196,072 & 11.9 & 68707 & 10685 & 39676 & 21277 & 33227 & 22500 \\
2010 & 1707441 & 201,370 & 11.8 & 63017 & 11530 & 40352 & 23881 & 38838 & 23752 \\
2011 & 1729934 & 207,547 & 12.0 & 70,175 & 12,586 & 32,557 & 21,747 & 42,934 & 27,548 \\
2012 & 1704915 & 172,838 & 10.1 & 63,286 & 12,905 & 23,370 & 22,346 & 27,007 & 23,924 \\
2013 & 1681077 & 149,418 & 8.9 & 57,115 & 12,184 & 20,330 & 20,712 & 20,831 & 18,246 \\
2014 & 1590331 & 132,895 & 8.4 & 54,174 & 10,587 & 16,637 & 17,966 & 15,558 & 17,973 \\
2015 & 1259014 & 100,877 & 8.0 & 39,267 & 8,016 & 13,671 & 11,647 & 12,022 & 16,254 \\
\hline
\end{tabular}

Fuente: elaboración propia con información del Secretariado Ejecutivo del Sistema Nacional de Seguridad Pública. Nota: la información para 2015 abarca hasta octubre. 
Cuadro A5

Distribución porcentual del número de robos en los estados de la frontera norte de México

\begin{tabular}{llllllll}
\hline Año & $\begin{array}{l}\text { Frontera } \\
\text { norte } \\
\text { (FN) }\end{array}$ & $\begin{array}{l}\text { Baja } \\
\text { California } \\
\%\end{array}$ & $\begin{array}{l}\text { Sonora } \\
\%\end{array}$ & $\begin{array}{l}\text { Chihuahua } \\
\%\end{array}$ & $\begin{array}{l}\text { Coahuila } \\
\%\end{array}$ & $\begin{array}{l}\text { Nuevo } \\
\text { León } \\
\%\end{array}$ & $\begin{array}{l}\text { Tamaulipas } \\
\%\end{array}$ \\
\hline 2005 & 149,694 & 34.6 & 8.8 & 20.9 & 6.3 & 15.5 & 13.9 \\
2006 & 165,792 & 35.9 & 7.5 & 21.2 & 6.0 & 16.0 & 13.5 \\
2007 & 187,013 & 38.9 & 7.7 & 15.7 & 6.2 & 19.0 & 12.4 \\
2008 & 203,260 & 36.9 & 5.9 & 19.3 & 8.5 & 17.8 & 11.6 \\
2009 & 196,072 & 35.0 & 5.4 & 20.2 & 10.9 & 16.9 & 11.5 \\
2010 & 201,370 & 31.3 & 5.7 & 20.0 & 11.9 & 19.3 & 11.8 \\
2011 & 207,547 & 33.8 & 6.1 & 15.7 & 10.5 & 20.7 & 13.3 \\
2012 & 172,838 & 36.6 & 7.5 & 13.5 & 12.9 & 15.6 & 13.8 \\
2013 & 149,418 & 38.2 & 8.2 & 13.6 & 13.9 & 13.9 & 12.2 \\
2014 & 132,895 & 40.8 & 8.0 & 12.5 & 13.5 & 11.7 & 13.5 \\
2015 & 100,877 & 38.9 & 7.9 & 13.6 & 11.5 & 11.9 & 16.1 \\
\hline
\end{tabular}

Fuente: elaboración propia con información del Secretariado Ejecutivo del Sistema Nacional de Seguridad Pública. Nota: la información para 2015 abarca hasta octubre. 
58 Ensayos Revista de Economía 